\title{
Active Vibration Control of Helicopter Maneuvering Flight Using Feedforward-Robust Hybrid Control Based on Reference Signal Reconstruction
}

\author{
Yifan Qin $(\mathbb{D}$, Yang Lu $\mathbb{D}$, Jinchao Ma $\mathbb{D}$, and Huiyu Yue $\mathbb{D}$ \\ National Key Laboratory of Rotorcraft Aeromechanics, College of Aerospace Engineering, \\ Nanjing University of Aeronautics and Astronautics, Nanjing 210016, China \\ Correspondence should be addressed to Yang Lu; njluyang@163.com
}

Received 30 June 2021; Revised 16 November 2021; Accepted 30 November 2021; Published 15 December 2021

Academic Editor: Jinxin Liu

Copyright (c) 2021 Yifan Qin et al. This is an open access article distributed under the Creative Commons Attribution License, which permits unrestricted use, distribution, and reproduction in any medium, provided the original work is properly cited.

Current control laws for active control of helicopter structural vibration are designed for steady-state flight conditions, while the vibration response of maneuvering flight has not been taken into consideration yet. In order to obtain full-time vibration suppression capability, the authors propose a filtered least mean square-mixed sensitivity robust control method based on reference signal reconstruction (LMS-MSRC), driving piezoelectric stack actuators to suppress helicopter structural vibration response in maneuvering flight. When feedback controller designed by $H_{\infty}$ theory is implemented, active damping is added on the secondary path to weaken the adverse effects of its sudden changes in maneuvering flight state. Furthermore, a reference signal reconstruction scheme is given concerning equivalent secondary path. In addition, the reconstruction accuracy, the convergence speed, stability, and global validity of the hybrid controller are analysed. Compared with multichannel Fx-LMS, numerical simulations of LMS-MSRC for vibration suppression are undertaken with a helicopter simplified finite element model under several typical flight conditions. Further experiments of real-time free-free beam vibration control are performed, driven by a stacked piezoelectric actuator. The instantaneous overshoot of measured response is $42 \%$ less than the peak value and its attenuation reaches $85 \%$ within $2.5 \mathrm{~s}$. Numerical and experimental results reveal that the proposed algorithm is practical for suppressing transient disturbance and multifrequency helicopter vibration response during maneuvering flight with faster convergence speed and better robustness.

\section{Introduction}

Excessive helicopter structural vibration imposes discomfort on passengers and crew members both physically and mentally. Rotor aerodynamic load is one of the main sources of fuselage vibration [1]. The alternating load and moment generated in the hub center are transmitted to the fuselage through the rotor control system and the gearbox, thereby causing harmonic vibration. In order to suppress the airframe vibration, a lot of researches have been carried out on the active helicopter vibration control and the active control of structural response (ACSR) system developed at present has been successfully applied to stereotyped helicopters, such as EH-101, S-92, EC225, and UH-60M [2-5]. Various active control algorithms have shown good performance in practice.
However, conventional helicopter ACSR systems are only applicable for the steady-state vibration components; little attention is paid to the active vibration suppression during maneuvering flight [2-5]. When a helicopter performs a large-overload maneuver, the alternating aerodynamic load of the rotor changes drastically in a short time and results in severe vibration $[6,7]$. Furthermore, flight tests demonstrate that the rotor hub torque changes transiently so as to produce additional uncertain disturbances, which are difficult to model and bring a nonnegligible impact on the fuselage vibration level $[7,8]$. Figure 1 shows measured forward flight speed and acceleration response of a $\mathrm{CH}-53 \mathrm{G}$ helicopter during maneuvering flight [9]. It can be seen that the vibration load of blade passing frequency changes drastically when the helicopter performs acceleration and deceleration maneuvers in $40 \mathrm{~s}-60 \mathrm{~s}$. In addition, 

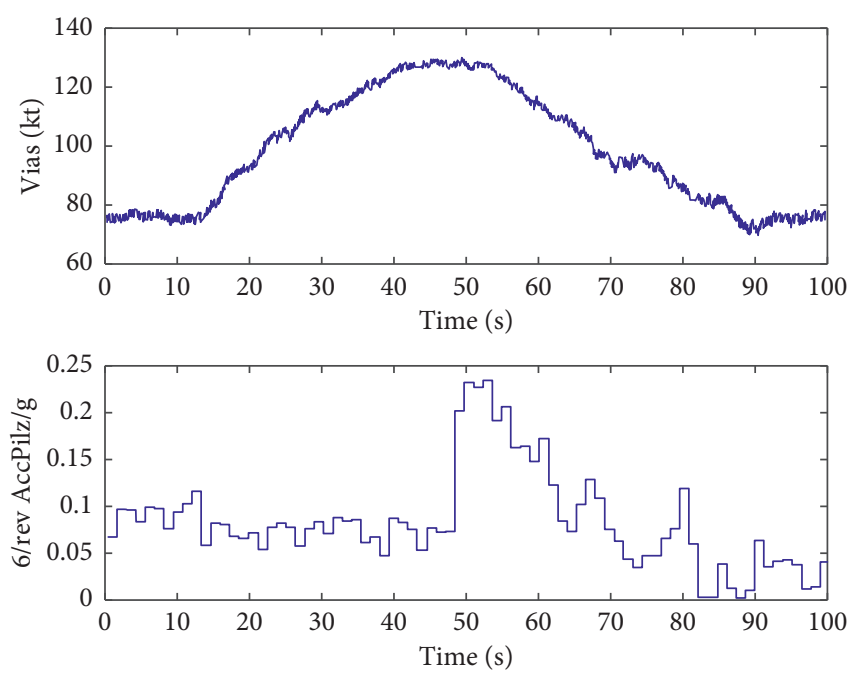

FIGURE 1: Measured acceleration response of a CH-53G helicopter during maneuvering flight.

instantaneous power demand changes in maneuvering flight also causes the rotor speed fluctuating significantly in a short time. Figure 2 illustrates vibration frequency oscillation when Bell 427 performed a left-turn maneuver [10]. The realtime vibration frequency may deviate from the target value $(22.4 \mathrm{~Hz})$ and can be reduced up to $5 \%$. On one hand, the control mechanism of the current ACSR systems limits the vibration suppression effects. On the other hand, the commonly used mechanical inertial actuators, such as electromagnetic actuators and circular force generators, have the shortcomings of narrow working frequency bands and slow response to instructions, respectively, which reduce the efficiency of ACSR systems severely $[4,11,12]$. For better adaptation to the working environment of helicopter maneuvering flight, research should be carried out on both actuator and control law design.

In recent years, piezoelectric stack actuator (PSA) has been widely used in helicopter active vibration control due to its advantages of large driving force, small mass and volume, wide frequency bandwidth, and fast response. Straub et al. used PSA to successfully realize the active vibration suppression of the full-scale SMART rotor [13]. Heverly et al. studied the working principle and distribution of PSAs based on the elastic finite element model of AH-64 scaled airframe comprehensively [14]. However, the characteristics of PSAs and their coupling effects with fuselage will change the actual secondary path in practice, imposing a negative impact on the active vibration suppression [14]. Viswamurthy and Ganguli pointed out that the modeling error of PSA could make it hard to predict the optimal input of the actual secondary path and weaken control performance [15]. To improve the control effects of PSAs in the helicopter ACSR during maneuvering flight, it is necessary to consider robustness to actual secondary path changes in the controller design.

Feedback control based on high-order harmonic control (HHC) and least mean square feedforward (Fx-LMS) control based on adaptive filtering technology are two control

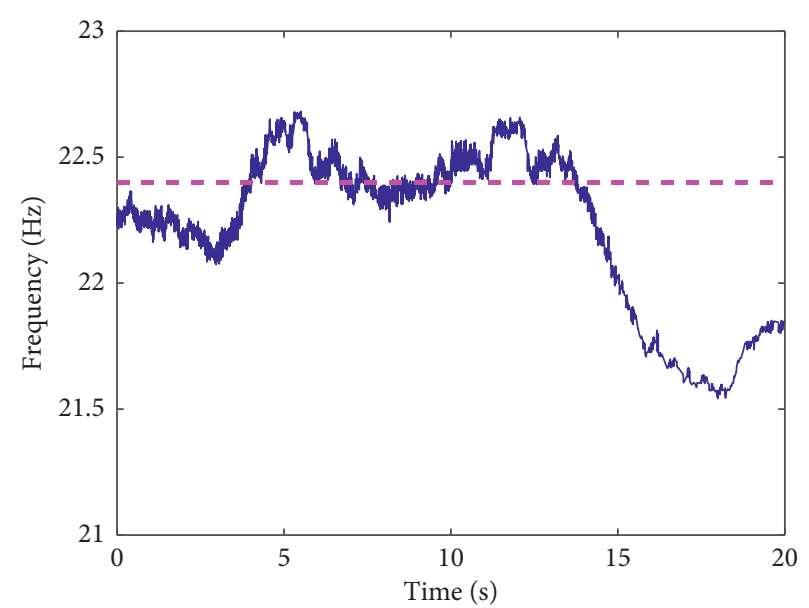

FIGURE 2: Oscillation in the vibration frequency of a Bell 427 during a left-turn maneuver.

methods widely used in helicopter $\operatorname{ACSR}[16,17]$. Based on linear and quasistatic assumptions, HHC can effectively suppress steady-state harmonic vibration in the frequency domain. But it is inevitable to perform discrete Fourier transform and inverse transform, $\mathrm{HHC}$ has a larger control interval and a slower update rate, making it suitable only for the situation where the external disturbance is relatively stable or secondary path changes barely [18]. In view of the shortcomings of traditional HHC, Zhao and Song used least mean square and recursive least square algorithm, respectively, to update harmonic parameters $[19,20]$. However, when there are frequency errors between the basis function and the excitations, the control system converges slowly [21]. Furthermore, the adverse impact of the secondary path mutation during maneuvering flight on vibration control is not considered.

Fx-LMS is an adaptive feedforward controller with good narrowband control effects. It shows strong signal tracking ability without requirement for accurate model and can be applied to the active vibration control of various helicopter flight states theoretically. However, the performance of Fx-LMS is directly influenced by the identification accuracy of secondary path and coherence between the reference signal and vibration signal [22]. The modeling error generated by secondary path mutation will decrease the adaptive ability of Fx-LMS and may even cause the control system to fail $[23,24]$. Moreover, for the vibration response with drastic changes in frequency and amplitude during maneuvering flight, with rotational speed signal used as reference signal directly, the coherence reduces so that the feedforward controller may not guarantee causal response and convergence speed will decrease [22, 25]. Considering the short duration of maneuver, it is necessary to find a new method with robustness enhancement and more accurate reference signal to enable the system to obtain stronger uncertain disturbance suppression performance.

For working out the problem that reference signal is in poor consistency with the uncontrolled response and the characteristics of the secondary path time vary, this paper 
proposes a filtered Least Mean Square-Mixed Sensitivity Robust Controller (LMS-MSRC) based on reconstructed reference signal, which drives PSAs to realize helicopter ACSR in maneuvering flight. The reference signal reconstructed by error signal can track the real-time changes of the uncontrolled response, which is beneficial for faster convergence speed. A feedback controller specially is designed by $H_{\infty}$ theory, and by the introduction of appropriate cost functions and weighting functions, the time-varying property of the secondary path is transformed into a robust performance design problem with uncertain perturbation output to solve, which enhances the robustness and convergence of the system.

The main contents of this paper are arranged as follows: the second section proposes specific improvement measures to establish the ACSR hybrid algorithm, where tracking accuracy of the reference signal reconstruction, system convergence and stability, and the global validity are analysed. A series of ACSR numerical simulations to verify the proposed controller in helicopter maneuvering flight status based on a simplified helicopter finite element model are presented in the third section. The fourth section carries out ACSR experimental research based on the free-free beam structure. Some conclusions are drawn from work of this paper in the last section.

\section{Feedforward-Robust Hybrid Algorithm Based on Reference Signal Reconstruction}

To better describe the proposed method, the classic Fx-LMS algorithm of helicopter ACSR is introduced and the deficiencies of its application in maneuvering flight are discussed. Furthermore, improvement measures are put forward to establish a hybrid control system and their advantages are analysed.

2.1. Classic Helicopter ACSR Fx-LMS Algorithm. As is shown in Figure 3, the reference signal $x(n)$ is a multifrequency signal related to the rotor excitation force, usually provided by the rotor speed. $\mathbf{P}(z)$ is the primary path that represents the physical transfer function between the rotor excitation and the error sensor at the controlled point (e.g., the pilot's position). $d(n)$ denotes the narrowband desired response. $d_{0}(n)$ is the transient multifrequency disturbance that is undetectable or hard to model. $\mathbf{S}(z)$ indicates the actual secondary path transfer function, including D/A, driving amplifier, actuator, dynamic characteristics between actuator and error sensor, filter, and $\mathrm{A} / \mathrm{D}$ converter. $x^{\prime}(n)$ is the filtered reference signal after $x(n)$ filtered by the secondary path offline estimation $\widehat{S}(z)$. $e(n)$ shows the error response at the controlled point. $\mathbf{W}(z)$ is an FIR filter of order $I$, which outputs the active control signal $y_{f}(k)$.

The error signal $e(n)$ is expressed as follows:

$$
e(n)=d(n)+d_{0}(n)-y(n)
$$

where $y(n)=\mathbf{S}(z) * \mathbf{Y}_{f}(n), \mathbf{Y}_{f}(n)=\left[y_{f}(n) \cdots y_{f}(n-J+1)\right]^{T}$.

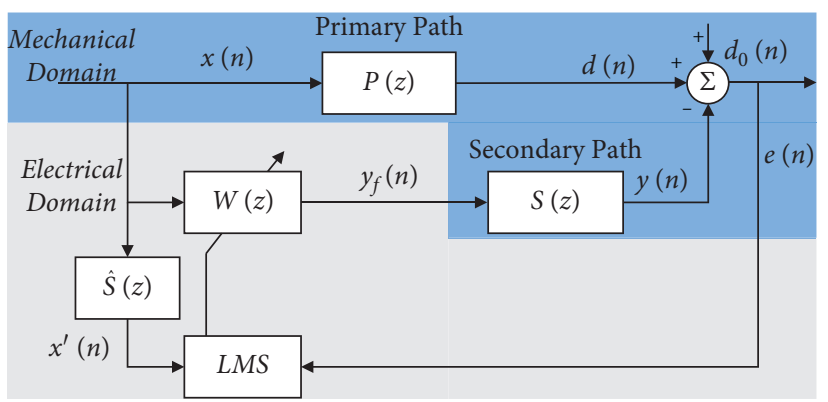

FIGURE 3: Block diagram of the classic helicopter ACSR Fx-LMS algorithm.

According to the steepest descent principle, the weight coefficient vector $\mathbf{w}(n)$ of $\mathbf{W}(z)$ is updated to adapt to realtime changes:

$$
\mathbf{w}(n+1)=\mathbf{w}(n)-2 \mu e(n) X^{\prime}(n),
$$

where $X^{\prime}(n)=\left[x^{\prime}(n) \cdots x^{\prime}(n-I+1)\right]^{T}, x^{\prime}(n)=\widehat{S}(z) *$ $\mathbf{X}(n)$, and $\mu$ is the step factor of control process. From equations (1)-(2), it is revealed that the reference signal $\mathbf{x}(n)$ and the actual secondary path $\mathbf{S}(z)$ play an important role in updating $\mathbf{w}(n)$. In helicopter maneuvering flight, $\mathbf{x}(n)$ hardly provides effective information about external excitation changes, leading to a decrease in system convergence. Moreover, the system stability problem caused by timevarying secondary path $\mathbf{S}(z)$ is not settled in maneuvering flight.

2.2. Proposed Algorithm. To overcome the above disadvantages, a feedforward-robust hybrid algorithm based on reference signal reconstruction is raised. The algorithm diagram is shown in Figure 4. For the purpose of robustness enhancement to the uncertainty of the secondary path, a feedback controller is designed based on the $H_{\infty}$ robust theory, which increases damping to improve stability and obtains overall vibration attenuation at the same time. Moreover, for the constructed equivalent secondary path, the reference signal is reconstructed based on the error signal, and the real-time excitation changes are fed back effectively to improve convergence performance. Detailed descriptions of the mixed sensitivity robust controller and reference signal reconstruction are as follows.

2.2.1. Mixed Sensitivity Robust Controller. The secondary path that changes drastically in a short time poses a threat to the stability of Fx-LMS during helicopter maneuvering flight. As is shown in Figure 5, the secondary path mutation $\Delta \mathbf{S}_{t}(z)$ during maneuvering flight is regarded as uncertain parameter perturbation, including modeling errors caused by helicopter dynamic changes and unmodeled dynamics in maneuvering flight. The design of mixed sensitivity robust controller is to convert the controller design problem of system perturbation into the robust performance design problem of uncertain parameter system. The robust stabilization problem is solved by selecting appropriate performance and weighting functions so as to obtain a suitable 


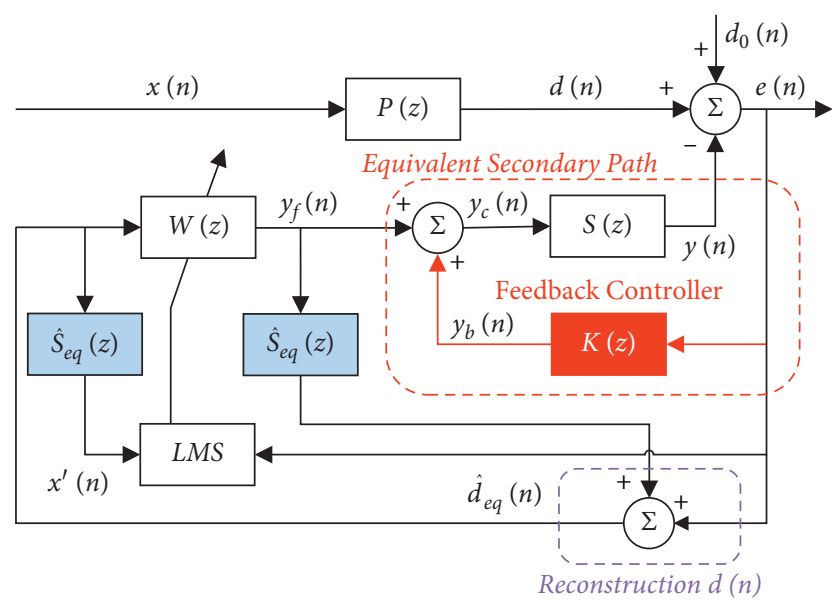

Figure 4: Sketch diagram of LMS-MSRC algorithm based on reference signal reconstruction. The red box describes the equivalent secondary path, and the purple one shows reconstruction of reference signal.

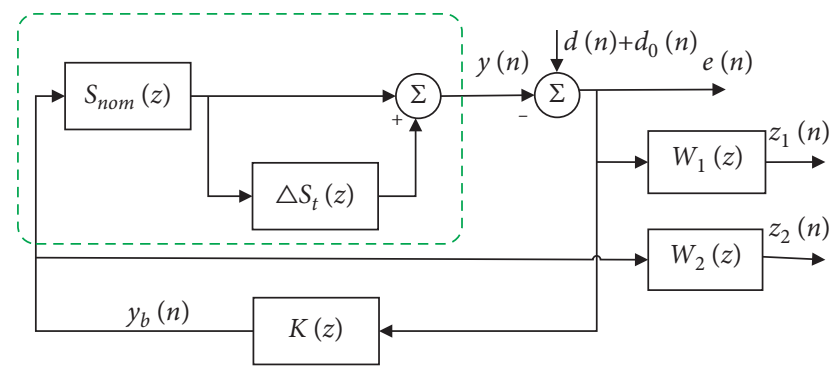

Figure 5: Hybrid sensitivity $H_{\infty}$ feedback control system based on multiplicative uncertainty.

controller, reducing the sensitivity to changes in secondary path and increasing the system damping simultaneously.

Multiplicative output uncertainty is used to represent the actual secondary path $\mathbf{S}(z)$. $\mathbf{S}_{\text {nom }}(z)$ denotes the nominal secondary path when $\Delta \mathbf{S}_{t}(z)=0$, which is generally identified by experiment. $\mathbf{K}(z)$ is the designed controller. $e(n)$, $y_{b}(n)$, and $d(n)+d_{0}(n)$ are error, control signal, and disturbance, respectively. $z_{1}(n)$ and $z_{2}(n)$ are the observation output. Therefore, the actual secondary path is

$$
\mathbf{S}(z)=\mathbf{S}_{\text {nom }}(z) \times\left[1+\Delta \mathbf{S}_{t}(z)\right] .
$$

According to [26], the control system is robust if it maintains stability and complies with certain performance criteria in the presence of uncertainty. As we can see from
Figure 5 , the transfer function from disturbance signal $d(n)+d_{0}(n)$ to $e(n)$ is

$$
\mathbf{H}_{e d}(z)=-[1+\mathbf{S}(z) \mathbf{K}(z)]^{-1} .
$$

If disturbance suppression performance of the closedloop system is required to be as good as possible to suppress the response perturbation of $d(n)+d_{0}(n)$ to $e(n)$ caused by secondary path change $\Delta \mathbf{S}_{t}(z), \mathbf{K}(z)$ needs to meet the following requirements [27]:

$$
\left\|\Delta \mathbf{S}_{t}(z) \times\left[1+\mathbf{S}_{\text {nom }}(z) \mathbf{K}(z)\right]^{-1}\right\|_{\infty} \leq 1 .
$$

Or in a strengthened form:

$$
\left\|\left[1+\mathbf{S}_{\text {nom }}(z) \mathbf{K}(z)\right]^{-1}\right\|_{\infty} \leq \frac{1}{\left\|\Delta \mathbf{S}_{t}(z)\right\|_{\infty}} .
$$

In the sense of $\infty$-norm, if it is necessary to maintain sufficient disturbance suppression performance for the largest possible perturbations $\Delta \mathbf{S}_{t}(z)$, we need to solve the following minimization problem to find $\mathbf{K}(z)$ :

$$
\min _{\mathbf{K} \text { stabilizing }}\left\|\left[1+\mathbf{S}_{\text {nom }}(z) \mathbf{K}(z)\right]^{-1}\right\|_{\infty} .
$$

In many cases, we may have a priori knowledge of the perturbation, and the perturbation block can be exhibited as follows:

$$
\Delta \mathbf{S}_{t}(z)=\widetilde{\Delta} \mathbf{S}_{t}(z) \mathbf{W}_{1}(z),
$$

where $\widetilde{\Delta} \mathbf{S}_{t}(z)$ is the unit norm perturbation set and weighting function. $\mathbf{W}_{1}(z)$ is designed to satisfy disturbance suppression demands. At this time, the suppression target is changed from $e(n)$ to $z_{1}(n)$. Correspondingly, the robust performance condition becomes

$$
\left\|\mathbf{W}_{1}(z)\left[1+\mathbf{S}_{\text {nom }}(z) \mathbf{K}(z)\right]^{-1}\right\|_{\infty} \leq \frac{1}{\left\|\widetilde{\Delta} \mathbf{S}_{t}(z)\right\|_{\infty}} .
$$

The optimization problem turns into

$$
\min _{\mathbf{K} \text { stabilizing }}\left\|\mathbf{W}_{1}(z)\left[1+\mathbf{S}_{\text {nom }}(z) \mathbf{K}(z)\right]^{-1}\right\|_{\infty} .
$$

In engineering practice, a combination of various cost functions should be included in a reasonable design. For example, disturbance suppression and limiting control signal energy are meaningful. As is illustrated in Figure 5, the above two goals are considered at the same time through mixed sensitivity controller design.

$$
\min _{\mathbf{K} \text { stabilizing }}\left\|\begin{array}{l}
\mathbf{W}_{1}(z) \times \mathbf{H}_{e d}(z) \\
\mathbf{W}_{2}(z) \times \mathbf{H}_{y d}(z)
\end{array}\right\|_{\infty}=\left\|\begin{array}{c}
\mathbf{W}_{1}(z)\left[1+\mathbf{S}_{\mathrm{nom}}(z) \mathbf{K}(z)\right]^{-1} \\
\mathbf{W}_{2}(z) \mathbf{K}(z)\left[1+\mathbf{S}_{\mathrm{nom}}(z) \mathbf{K}(z)\right]^{-1}
\end{array}\right\|_{\infty},
$$

where $\mathbf{H}_{y d}(z)=\mathbf{K}(z) \times[1+\mathbf{S}(z) \mathbf{K}(z)]^{-1}$ represents the transfer function from disturbance signal $d(n)+d_{0}(n)$ to control signal $y_{b}(n)$.
2.2.2. Reference Signal Reconstruction. To solve the problem that the speed signal is difficult to reflect the real-time changes of disturbance amplitude, it is a better choice to 
directly use the feedforward component of the uncontrolled response $d(n)+d_{0}(n)$ to update $\mathbf{W}(z)$. However, since the actuator works continuously, it is hard to obtain the $d(n)+$ $d_{0}(n)$ from the control system in real time. At this moment, the feedback neutralization method can be used to obtain the reconstructed reference signal based on the error signal [28]. The reconstruction process is shown in Figure 4.

$$
\mathbf{S}_{e q}(z)=\frac{\mathbf{S}(z)}{1+\mathbf{S}(z) \mathbf{K}(z)},
$$

$\widehat{S}_{e q}(z)$ is a FIR filter of the equivalent secondary path $\mathbf{S}_{e q}(z)$, which is identified offline, and its order is $J$. Through $e(n)$ and $\mathbf{Y}_{f}(n)$ obtained in real time, the reference signal reconstruction process is

$$
\widehat{d}_{e q}(n)=e(n)+\widehat{S}_{e q}(n) * \mathbf{Y}_{f}(n),
$$

where $\widehat{d}_{e q}(n)$ is the reconstructed reference signal, representing the component that needs to be suppressed by feedforward control, that is, the vibration response only under the action of the robust feedback controller $\mathbf{K}(z)$.

The proposed hybrid control law can be summarized as follows:

$$
\left\{\begin{array}{l}
y_{f}(n)=\mathbf{w}(n) * \widehat{D}_{e q}(n), \\
y_{c}(n)=y_{f}(n)+y_{b}(n), \\
e(n)=d(n)+d_{0}(n)-\mathbf{s}(n) * \mathbf{Y}_{c}(n), \\
\widehat{d}_{e q}(n)=e(n)+\widehat{s}_{e q}(n) * \mathbf{Y}_{f}(n), \\
x^{\prime}(n)=\widehat{s}_{e q}(n) * \widehat{D}_{e q}(n), \\
\mathbf{w}(n+1)=\mathbf{w}(n)+2 \mu e(n) \widehat{D}_{e q}(n) .
\end{array}\right.
$$

2.3. Performance Analysis. To satisfy the requirements of helicopter ACSR in maneuvering flight, the advantages of the proposed hybrid system in terms of reference signal reconstruction accuracy, control system convergence and stability, and global effectiveness are analysed.

2.3.1. Reference Signal Reconstruction Accuracy. It can be seen from equation (13) that the reconstruction error of $\widehat{d}_{e q}(n)$ mainly depends on the modeling error between the offline identification of the equivalent secondary path and the actual. When $\mathbf{S}(z)$ changes during maneuvering flight, the equivalent secondary path $\mathbf{S}_{e q}(n)$ is expressed as equation (12). Assuming the offline identification of $\mathbf{S}(z)$ is $\widehat{S}(z)=\mathbf{S}_{\text {nom }}(z)$, the error compared to $\mathbf{S}(z)$ is

$$
\Delta \widehat{S}(z)=\mathbf{S}(z)-\widehat{S}(z)=\mathbf{S}_{\text {nom }}(z) \Delta \mathbf{S}_{t}(z) .
$$

Converting equation (15) in the form of sensitivity, we get

$$
\frac{\Delta \widehat{S}(z)}{\widehat{S}(z)}=\Delta \mathbf{S}_{t}(z)
$$

When $S_{t}(z) \neq 0$, the reconstruction error of the reference signal increases, and the hybrid control may diverge. With the robust feedback controller $\mathbf{K}(z)$ introduced, the increment of equivalent secondary path $\mathbf{S}_{e q}(n)$ estimated offline is as follows:

$$
\Delta \widehat{S}_{e q}(z)=\frac{\mathbf{S}_{\text {nom }}(z)\left[1+\Delta \mathbf{S}_{t}(z)\right]}{1+\mathbf{S}_{\text {nom }}(z)\left[1+\Delta \mathbf{S}_{t}(z)\right] \mathbf{K}(z)}-\frac{\mathbf{S}_{\text {nom }}(z)}{1+\mathbf{S}_{\text {nom }}(z) \mathbf{K}(z)} .
$$

Converting equation (17) into the form of sensitivity, we get

$$
\frac{\Delta \widehat{S}_{e q}(z)}{\widehat{S}_{e q}(z)}=\Delta \mathbf{S}_{t}(z) \mathbf{G}(z),
$$

where

$$
\mathbf{G}(z)=\frac{1}{1+\widehat{S}_{e q}(n) \mathbf{K}(z)} .
$$

When $\left|\widehat{S}_{e q}(z) \mathbf{K}(z)\right| \gg 1$, then $|\mathbf{G}(z)| \ll 1$. Compared with the case without $\mathbf{K}(z)$, the influence of the model error $\Delta \widehat{S}_{e q}(z)$ on $\widehat{S}_{e q}(z)$ declines at this time. In terms of the timevarying secondary path, the reference signal reconstruction error is reduced, which is beneficial to effectively track the changes of the external disturbance response, and the stability of the hybrid control system is enhanced.

2.3.2. Convergence and Stability Analysis. As is illustrated in equation (14), according to the reconstructed reference signal $\widehat{d}_{e q}(n)$, the recursive formula of the filter coefficient $\mathbf{w}(n)$ turns into

$$
\mathbf{w}(n+1)=\mathbf{w}(n)+2 \mu e(n) \widehat{D}_{e q}(n) .
$$

Compared to speed signal, $\widehat{d}_{e q}(n)$ enhances the coherence between reference signal and uncontrolled responses, which means that $\mathbf{w}(n)$ needs to pay less adjustment cost to adapt to drastically changing response during maneuvering flight, leading to faster convergence of the control system.

Furthermore, according to Diniz [29], the stability condition of Fx-LMS algorithm is 


$$
0<\mu<\frac{1}{\operatorname{tr}(\mathbf{R})}
$$

where $\mathbf{R}$ is the autocorrelation matrix of filtered reference signal and $\operatorname{tr}(\mathbf{R})$ is its trace, which satisfies

$$
\begin{aligned}
\mathbf{R} & =E\left[\widehat{D}_{e q}(n) \widehat{D}_{e q}^{T}(n)\right] \\
& =\left[\begin{array}{cccc}
\widehat{d}_{e q}^{2}(n) & \hat{d}_{e q}(n) d_{e q}^{\prime}(n-1) & \cdots & \widehat{d}_{e q}(n) \widehat{d}_{e q}(n-I+1) \\
\hat{d}_{e q}(n-1) d_{e q}^{\prime}(n) & \hat{d}_{e q}^{2}(n-1) & \cdots & \widehat{d}_{e q}(n-1) \widehat{d}_{e q}(n-I+1) \\
\vdots & \vdots & \ddots & \vdots \\
\hat{d}_{e q}(n-I+1) d_{e q}(n) & \hat{d}_{e q}(n-I+1) d_{e q}(n-1) & \cdots & \hat{d}_{e q}^{2}(n-I+1)
\end{array}\right], \\
\operatorname{tr}(\mathbf{R}) & =\sum_{i=0}^{I-1} \hat{d}_{e q}^{2}(i) .
\end{aligned}
$$

According to equations (21)-(23), if $\mathbf{S}(z)$ dynamics is low-damped and the impulse response of $x^{\prime}(n)$ is large with long duration, $\operatorname{tr}(\mathbf{R})$ will increase and the upper limit of $\mu$ will decrease, making system convergence and stability reduce. However, after the robust controller $\mathbf{K}(z)$ is introduced, $\widehat{S}(z)$ turns into the offline estimation $\widehat{S}_{e q}(z)$ of the equivalent secondary path $\mathbf{S}(z) / 1+\mathbf{S}(z) \mathbf{K}(z)$. Owing to $1+S(z) K(z) \gg 1,\left|\widehat{S}_{e q}(z)\right| \ll|\widehat{S}(z)|$, then the impulse response of $\widehat{d}_{e q}(n)$ is small and short, contributing to small $\operatorname{tr}(\mathbf{R})$ and little $\mu$. In fact, $\mathbf{S}_{e q}(z)$ provides higher damping than $\mathbf{S}(z)$, which is beneficial for convergence and stability enhancement.

2.3.3. Global Validity. From Figure 4, the vibration response after control is

$$
E(z)=\frac{1}{1+\mathbf{S}(z) \mathbf{K}(z)}\left[D(z)+D_{0}(z)\right]+\frac{\mathbf{S}(z) \mathbf{W}(z)}{1+\mathbf{S}(z) \mathbf{K}(z)} \widehat{D}_{e q}(z)
$$

where $E(z), D(z), D_{0}(z)$, and $\widehat{D}_{e q}(z)$ denote the $z$-transforms of $e(n), d(n), d_{0}(n)$, and $\widehat{d}_{e q}(n)$. If designed $\mathbf{K}(z)$ can ensure system stability and satisfy $1+S(z) K(z) \gg 1$ in the target frequency band, for the drastically changing desired signal $d(n)+d_{0}(k)$ under maneuvering flight conditions, the feedback loop can still control it to a small level (but not to 0 ), even if $W(z)=0$. If the filter is adjusted to $\mathbf{W}(z)=-\mathbf{S}^{-1}(z)\left[D(z)+D_{0}(z)\right] \widehat{D}_{e q}^{-1}(z)$, global vibration reduction can be achieved theoretically. Therefore, the hybrid control system indicates advantages over either independent feedback or feedforward control when there is transient disturbance: the former is responsible for overall response reduction, while the latter helps to offset the vibration residuals. The complementarity is effective for realizing vibration control of maneuvering flying helicopters.

\section{Simulation Results and Analysis}

To verify the effectiveness of the LMS-MSRC control law based on reference signal reconstruction, a single-input single-output control system is constructed to carry out ACSR simulation research of the helicopter maneuver flight, which is based on the secondary path identified offline of the simplified helicopter finite element model. The simulations of conventional ACSR methods are carried out simultaneously for comparison so as to discuss the advantages of the proposed method in control effect, stability, and robustness with complex external disturbances and structural parameter changes during maneuvering flight.

3.1. Simulation Model. Currently, the elastic beam structure model has been widely used in helicopter vibration response analysis [30]. As is shown in Figure 6, a free-free finite element beam model built by ANSYS is regarded as the simplified helicopter fuselage [31]. The structure parameters are as follows: $m=6.1 \times 10^{3} \mathrm{~kg}, \rho=7.8 \times 10^{3} \mathrm{~kg} / \mathrm{m}^{3}$, $E=210 \mathrm{Gpa}$, and $\mu=0.3$. Among them, $m, \rho, E$, and $\mu$ represent mass, density modulus of elasticity, and Poisson's ratio, respectively. The first seven modes are analysed as 8.2, $11.2,16.7,26.9,33.2,50.4$, and $56.9 \mathrm{~Hz}$. Figure 6(b) displays a partial enlargement of front part of the fuselage. External disturbance is applied at node 94 to simulate excitation force from the hub, and secondary control force is introduced at node 109 . The 37 th node represents the driver's seat, and its acceleration response is used to evaluate the global effectiveness of the active control system.

Figure 7 indicates the measured acceleration response power spectrum of a typical helicopter [19]. To simulate the amplitude and frequency characteristics of the helicopter vibration response, the blade passing frequency is set to $19.5 \mathrm{~Hz}$ with second-order harmonic set to $39 \mathrm{~Hz}$. By adjusting disturbance amplitude, the vibration response of $19.5 \mathrm{~Hz}$ is about $12 \mathrm{~dB}$ larger than the second-order harmonic component at node 37 . Gaussian white noise with 0 mean value is added to the multifrequency signal.

3.2. Design of the Robust Feedback Controller. The performance function $\mathbf{W}_{1}(z)$ is related to the excitation load by 

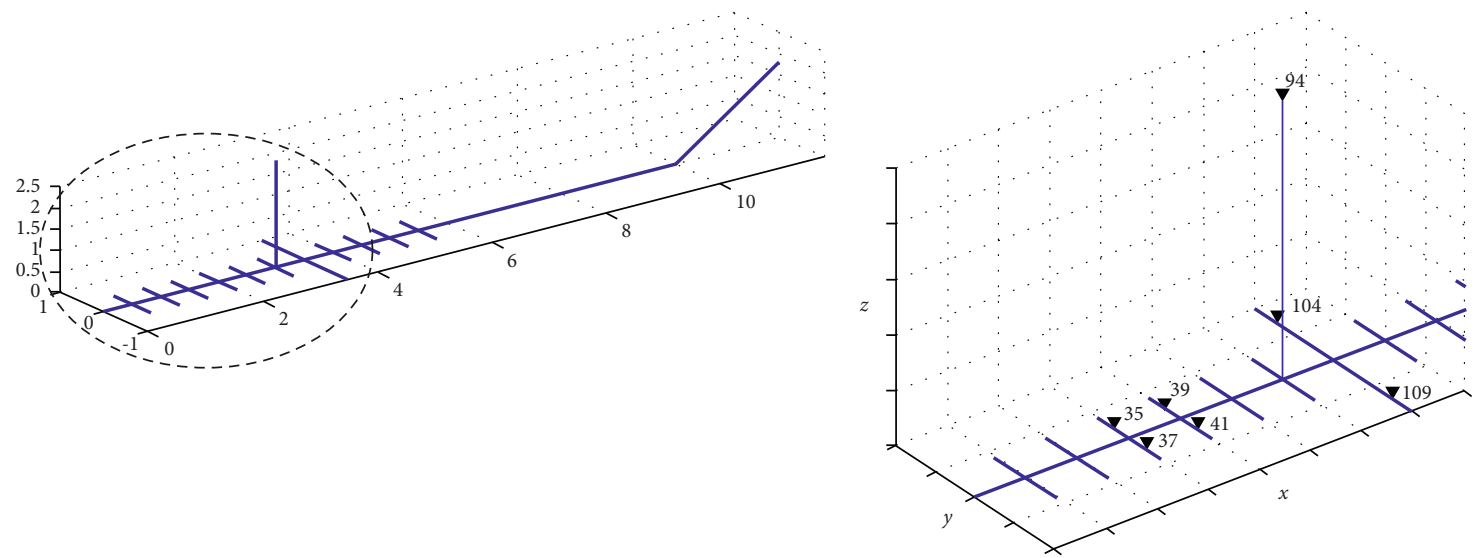

(a)

(b)

Figure 6: Simplified finite element model of the helicopter fuselage. (a) The whole fuselage. (b) Front part of the fuselage.

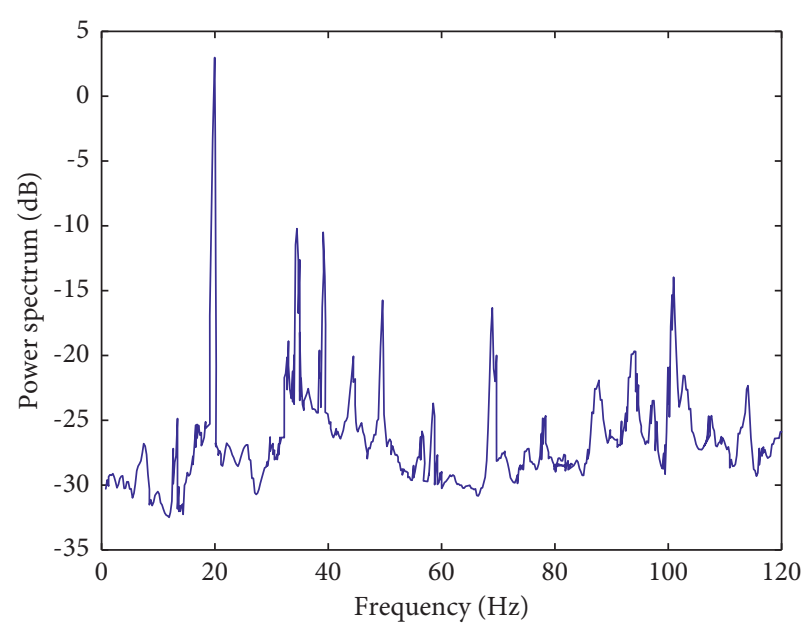

Figure 7: Measured acceleration response power spectrum of a typical helicopter.

helicopter, and it needs a large amplitude at the disturbance frequencies. Taking into consideration time-varying transfer functions during maneuvering, the design margin of the controller can be increased and the parameter perturbation range of the nominal secondary path can be set to $20 \%$. Moreover, for the sake of multifrequency characteristics of disturbance and avoiding system natural frequencies to reduce complexity, $\mathbf{W}_{1}(z)$ is designed as a narrowband pass filter with excitation frequencies, as shown in equation (25). $\lambda, f_{w i}$, and $\sigma_{i}$ reveal adjustment coefficient, disturbance frequency, and system damping ratio separately. $\mathbf{W}_{2}(z)$ is used to limit input amplitude of the actuator and can be set as a constant gain 1 .

$$
W_{1}(s)=\lambda \prod_{i=1}^{n} \frac{f_{w i}^{2}}{s^{2}+2 f_{w i} \sigma_{i} s+f_{w i}^{2}} .
$$

With proper weighting functions and MATLAB robust control toolbox, the robust feedback controller is designed as a 10 -order $z$-domain controller.
3.3. Numerical Results and Analysis. It is worth noting that the environment of helicopter maneuvering flight is very complicated, which is caused by time-varying secondary path, drastic changes of external disturbance, and so on. Firstly, the accuracy of the reference signal reconstruction after secondary path mutation is analysed. Furthermore, the robust feedback controller's ability to suppress transient external disturbance and secondary channel mutation is verified. In addition, a simulation is carried out for timevarying disturbance amplitude and frequency. At last, all the above factors are considered simultaneously to check the performance of simulated helicopter maneuvering flight.

3.3.1. Reference Signal Reconstruction. To confirm the accuracy of the reconstructed reference signal, the original and reconstructed reference signals after secondary path mutation are exhibited in Figure 8. The amplitude-frequency characteristic of the secondary path is magnified by a factor of 1.3 , and the damping is slightly reduced. Extra disturbance is applied at $15 \mathrm{~s}$, and all responses are normalized by the peak value of the original reference signal before interference. As is shown in Figure 8, the reconstructed reference signal shows good consistency with the original, and the overall error is less than $10.2 \%$ after mutation. Although the error oscillates after $15 \mathrm{~s}$, it does not show a tendency to diverge, which proves the reconstructed signal good tracking abilities to change to the original. It is found from the subsequent simulation that the error to this extent is acceptable and the hybrid control system works well.

\subsubsection{Secondary Path Mutation and Transient External} Disturbance. In order to confirm the benefits of the proposed LMS-MSRC to the robustness of ACSR system in maneuvering flight, four helicopter ACSR methods are compared in Figure 9. The employed ACSR methods for comparison including enhanced HHC, frequency-domain LMS, and multichannel LMS are wildly studied in the literature $[19,21,23]$. The parameters in all the methods are optimized after several attempts. The control starts to be 


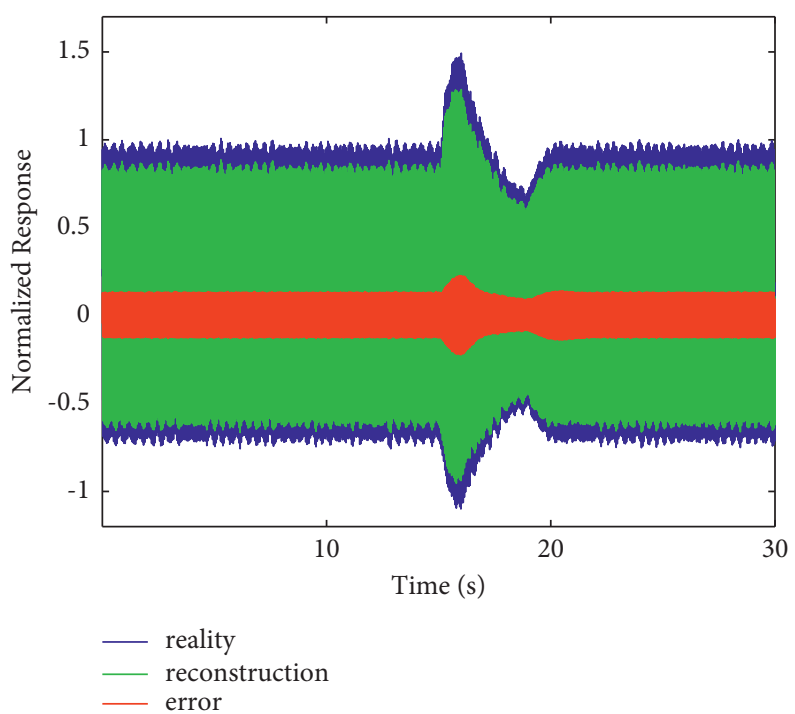

FIgURE 8: The original and reconstructed reference signal after secondary path mutation.

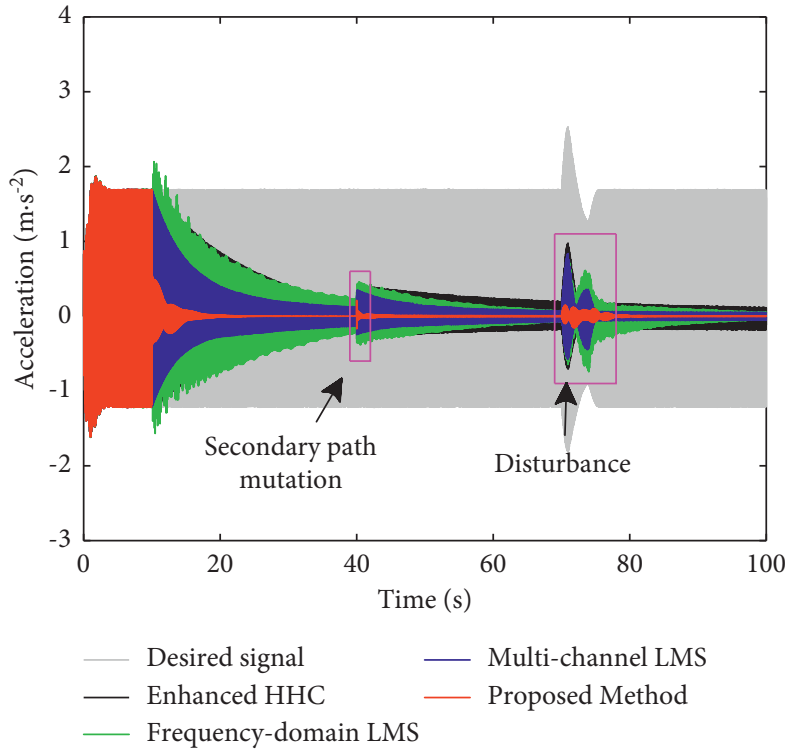

(a)
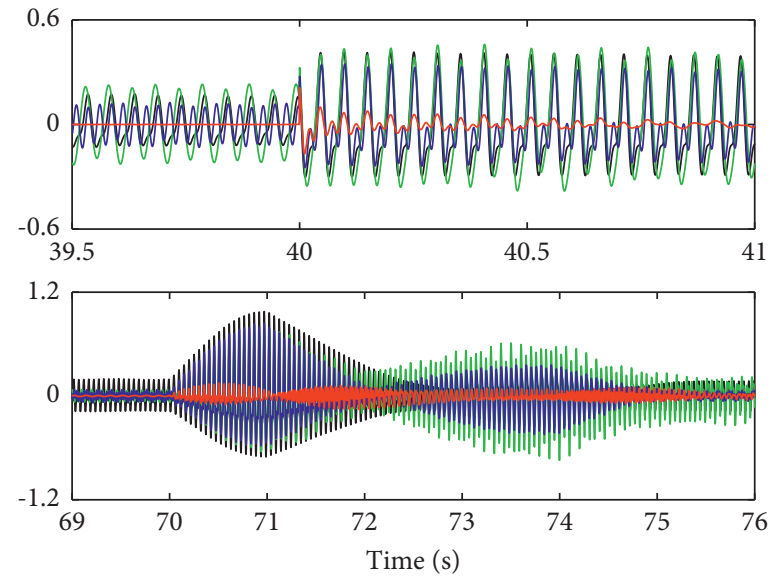

(b)

FIgURE 9: Comparison of acceleration response with different helicopter ACSR methods. (a) Acceleration response at the control point. (b) Control process at mutation and disturbance.

exerted 10s after the external disturbance. In order to simulate secondary path mutation and transient disturbance that are hard to model during maneuvering, the amplitudefrequency characteristic of the secondary channel is decreased by $15 \%$ with damping slightly reduced at $40 \mathrm{~s}$, and additional disturbance is applied at $70 \mathrm{~s}$. The comparison of acceleration response with different methods is displayed in Figure 9.

Considering enhanced HHC, the convergence speed after secondary path mutation is slower than that of other methods and the transient external disturbance that is difficult to model can hardly be suppressed. Secondary path mutation adaptability is improved by frequency-domain
LMS, while residual response oscillation is more severe under the circumstances of injected disturbance. In contrast, control effects in multichannel LMS and proposed LMSMSRC are relatively more effective. It takes $30 \mathrm{~s}$ for multichannel LMS to achieve $93 \%$ of vibration attenuation, while LMS-MSRC converges quickly, and the acceleration response is almost suppressed completely after $8 \mathrm{~s}$. Both control laws can adapt to transfer function changes, but the convergence is decreased with multichannel LMS. Owing to the lack of necessary reference information, multichannel LMS shows poor adaptability to interference changes. On the contrary, the vibration contributed by transient disturbance is attenuated over $90 \%$ by LMS-MSRC. It proves 
that the robust feedback loop can compensate secondary path and perform a stronger suppression performance on system parameter perturbation and uncertain transient disturbance, which is beneficial for robustness and effectiveness improvement of helicopter maneuvering ACSR system. Subsequent simulations and experiments are carried out with multichannel LMS and LMS-MSRC for consideration of application potential.

3.3.3. Time-Varying Excitation Amplitude and Frequency. The system is excited by $19.5 \mathrm{~Hz}+39 \mathrm{~Hz}$ external disturbance and control begins after 10s. Taking into account actual working conditions of forward flight to speed up [8], excitation signal amplitude increases by $50 \%$ every $8 \mathrm{~s}$ starting from $40 \mathrm{~s}$ and returns to initial value at $64 \mathrm{~s}$. The disturbance frequencies are reduced by $5 \%$ (turn to $18.5 \mathrm{~Hz}+37 \mathrm{~Hz}$ ) to reappear rotor speed reduction during maneuvering flight at $80 \mathrm{~s}$. The parameter settings remain unchanged. The filter order is set to 64 , and the initial value of the weight coefficients is 0 . The system sampling rate is $1000 \mathrm{~Hz}$. Convergence factors of multichannel LMS are $\mu_{19.5 \mathrm{~Hz}}=6 \times 10^{-4}, \mu_{39 \mathrm{~Hz}}=1.5 \times 10^{-3}$ while the value of LMS-MSRC is $\mu=6 \times 10^{-2}$. The adjustment coefficients, external disturbance frequencies, and system damping ratio are designed as follows: $\lambda=0.2, f_{w i}=2 \pi \times[19.5,39]$, and $\sigma_{i}=[0.06,0.025]$. The vibration control process is displayed in Figures 10 and 11.

The increase in excitation amplitude requires more control energy, which is reflected in the enlargement in filter output shown in Figures 10(b) and 11(b). For multichannel LMS in Figure 10(b), it is observed that the transient increment of filter output is almost close to zero at the beginning of mutation, indicating that the algorithm converges slowly. Comparing Figure 10(a) with Figure 11(a), it is found that multichannel LMS tends to seek out stability at the cost of convergence in the circumstances of amplitude surge. The reasons accounting for this phenomenon are that convergence factors affect each other when dealing with multifrequency components. On the other hand, the speed signal fails to provide real-time changes in disturbance, resulting in additional costs to fit system changes. In contrast, the reconstructed reference signal in LMS-MSRC can effectively track excitation changes and the convergence speed is accelerated under the premise of stability.

3.3.4. Complex Operating Conditions in Helicopter Maneuvering Flight. All the above factors are taken into account to simulate complex situation of helicopter maneuvering ACSR [8]. Vibration is suppressed before $10 \mathrm{~s}$. With the magnitude-frequency characteristic of secondary path multiplied by 0.85 and damping slightly reduced, excitation amplitude increases by $50 \%$ and additional transient disturbance is applied. At the same time, excitation frequency is reduced by $5 \%$ to simulate the decrease in rotor speed. The parameter settings remain unchanged, and the control processes are shown in Figures 12 and 13, respectively.

It is difficult for multichannel LMS to adapt to complex external disturbance and secondary path changes. The vibration overshoot exceeds the peak value of uncontrolled response. The filter output regulates slowly so that residual vibration still reaches $15 \%$ after 20 s. LMS-MSRC shows good adaptability by contrast. The peak response of closedloop system is $60 \%$ less than the uncontrolled response, and acceleration response is decreased by $95 \%$ within $3.5 \mathrm{~s}$. Therefore, the proposed hybrid algorithm can suit structural parameter changes and transient response via a robust controller and accelerate convergence according to the reconstructed reference signal reflecting the real-time changes of external disturbance. The results demonstrate that LMS-MSRC is superior to multichannel LMS in terms of vibration response suppression on complex maneuvering conditions.

Summarizing the above simulation results, it is not difficult to draw the conclusions: compared with multichannel LMS, LMS-MSRC based on reference signal reconstruction shows stronger adaptive ability with better robustness and faster convergence. It indicates great vibration suppression capability for complicated operating conditions of helicopter maneuvering flight.

\section{Active Control Tests and Results Analysis}

In order to verify the effectiveness of the LMS-MSRC algorithm, considering actual demands of helicopter vibration reduction and the scale of the control system, a series of ACSR tests based on the rotor passing frequency and its second-order frequency component $(19.5 \mathrm{~Hz}+39 \mathrm{~Hz})$ are carried out.

4.1. Experimental System Setup. The block diagram of the elastic beam ACSR test system is shown in Figure 14. The beam is suspended by steel cables to realize free-free boundary conditions. Excitation signal generated by the signal generator (1000Z, DG) drives the exciter (HEA-500, SinoCera) via the power amplifier to excite the elastic beam to produce multifrequency vibration. The uncontrolled vibration is monitored by the acceleration sensor (333B30, PCB), low-pass filtered by the signal conditioner (CM3504, Centuryl), and enters into the data acquisition system (DH5902, Donghua). According to the active control law, real-time control signal calculated by DSP drives the PSA to generate the required control torque via the driving power, neutralizing the response caused by external excitation. The ACSR physical testing system is displayed in Figure 15. Specific parameters of the beam structure and the lowvoltage mechanically packaged PSA used in this test are exhibited in Table 1 and Table 2, respectively.

4.2. Experimental Results and Analysis. The performance tests of the proposed controller are carried out as below: firstly, a dual-frequency harmonic excitation control experiment is conducted. Then, the adaptability of LMSMSRC with time-varying phases is verified. The control experiment about simultaneous changes in excitation frequency and amplitude as well as primary/secondary path mutation is implemented. The convergence factors are 


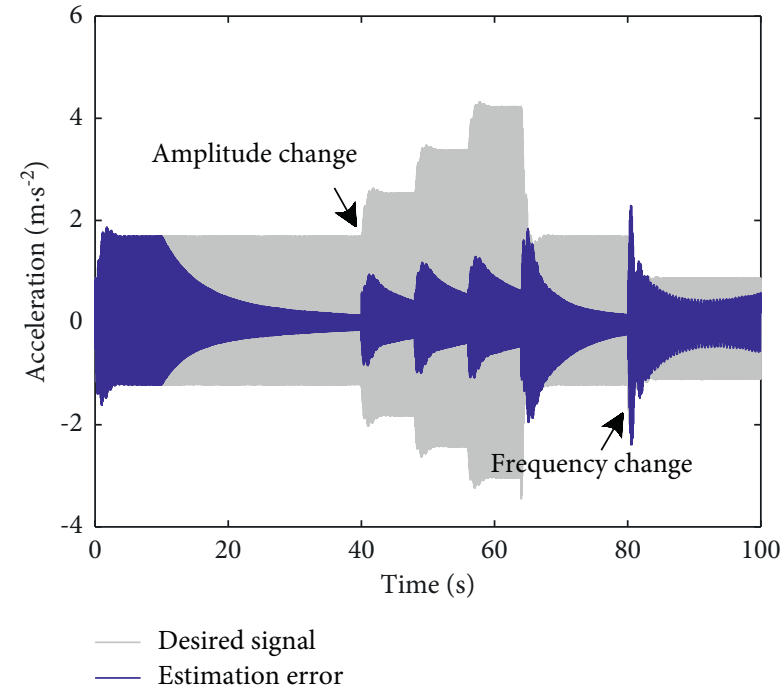

(a)

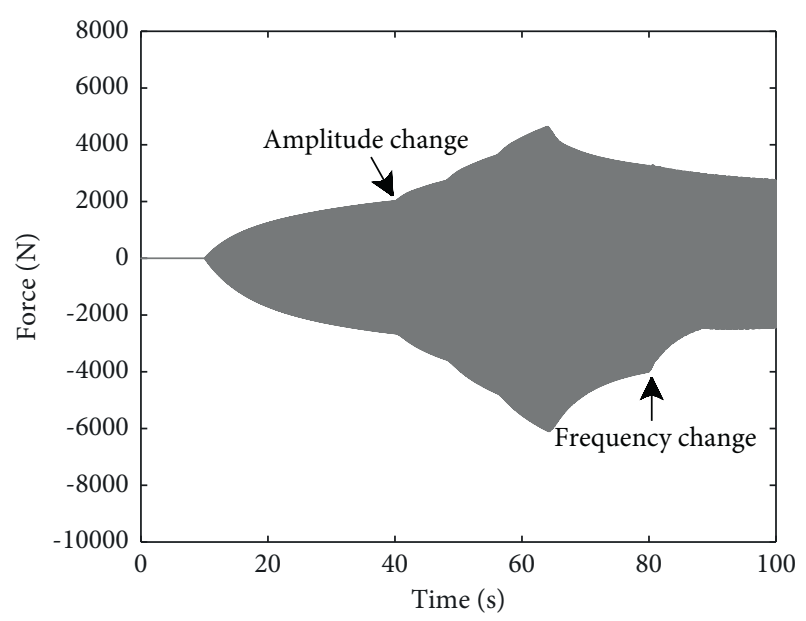

(b)

FIGURE 10: Acceleration response and filter output with multichannel LMS algorithm. (a) Acceleration response at the control point. (b) Output of the filter.

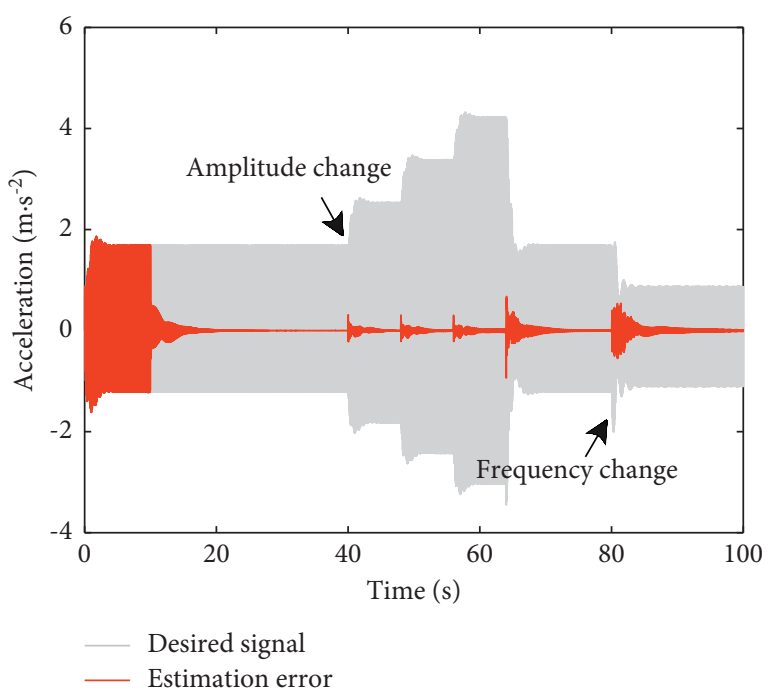

(a)

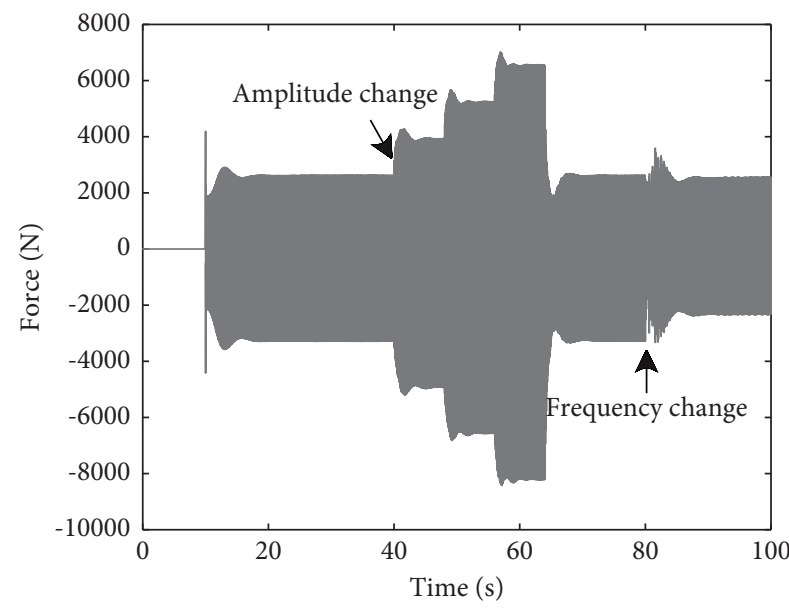

(b)

FIGURE 11: Acceleration response and filter output with LMS-MSRC algorithm. (a) Acceleration response at the control point. (b) Output of the filter.

$\mu_{19.5 \mathrm{~Hz}}=8 \times 10^{-4}, \mu_{39 \mathrm{~Hz}}=1 \times 10^{-3}$ for multichannel LMS and $\mu=2 \times 10^{-3}$ for LMS-MSRC. The sampling rate is $1000 \mathrm{~Hz}$. The filter coefficient is 64 , and the initial value is set to 0 . The adjustment coefficient $\lambda$, external disturbance frequency $f_{w i}$, and system damping ratio $\sigma_{i}$ are designed as follows: $\lambda=0.25, f_{w i}=2 \pi \times[19.5,39]$, and $\sigma_{i}=[0.08,0.035]$.

4.2.1. Dual-Frequency Harmonic Excitation. Control starts at 10s. The error response at the controlled position and the filter output are exhibited in Figure 16. It can be seen that the acceleration response quickly drops below $12 \%$ after control, while it takes at least 30s for multichannel LMS to converge. After the system is stabilized, vibration of target frequencies is almost completely eliminated, which indicates the designed LMS-MSRC can suppress effective vibration level at the position of helicopter fuselage. Figure 16(d) shows the filter output with LMS-MSRC.

4.2.2. Harmonic Disturbance with Time-Varying Phase. By adjusting the phase of the driving signal input to the exciter, harmonic excitation phase changes from $0^{\circ}$ to $+45^{\circ}$, $-30^{\circ}$ and $0^{\circ}$ at $80 \mathrm{~s}, 160 \mathrm{~s}$, and $240 \mathrm{~s}$, respectively. The control process is shown in Figure 17. The residual vibration 


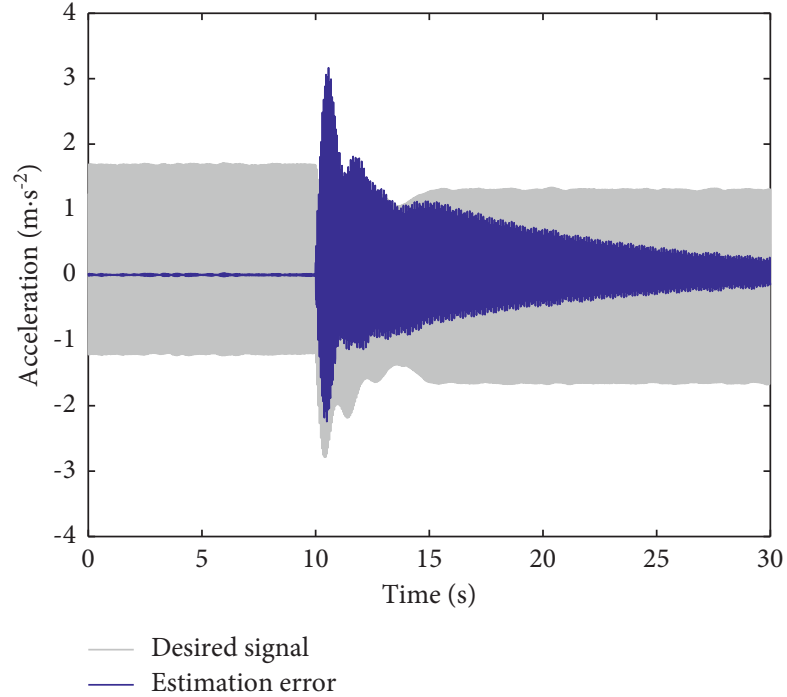

(a)

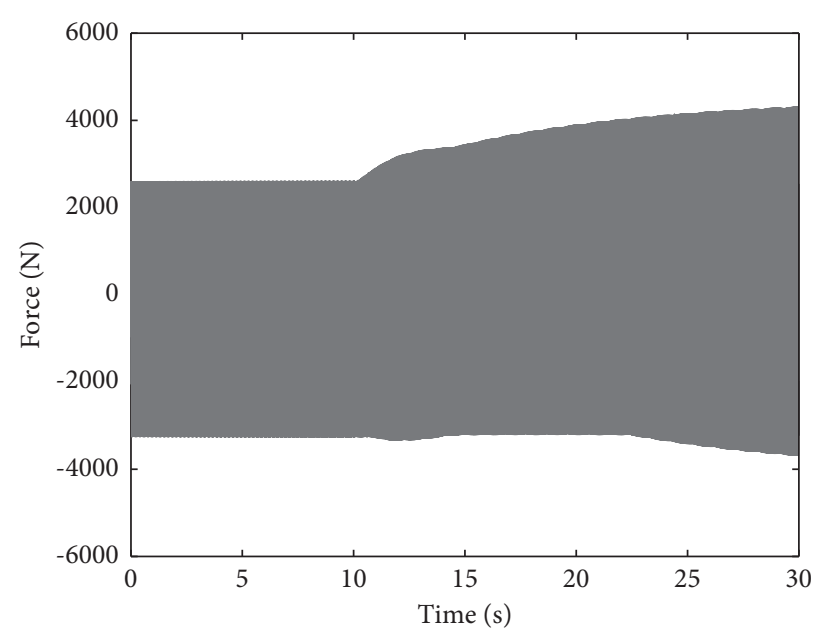

(b)

FIGURE 12: Acceleration response and filter output with multichannel LMS algorithm. (a) Acceleration response at the control point. (b) Output of the filter.

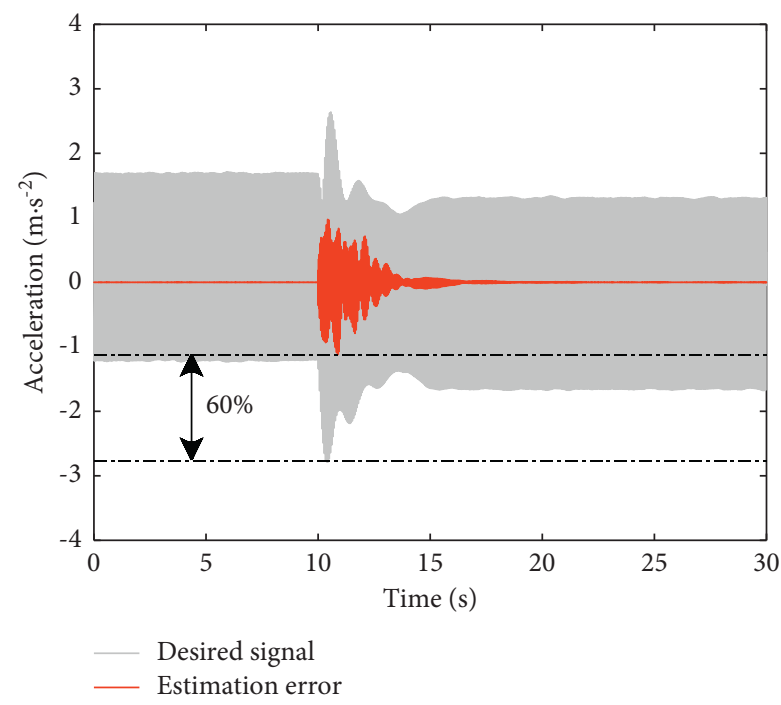

(a)

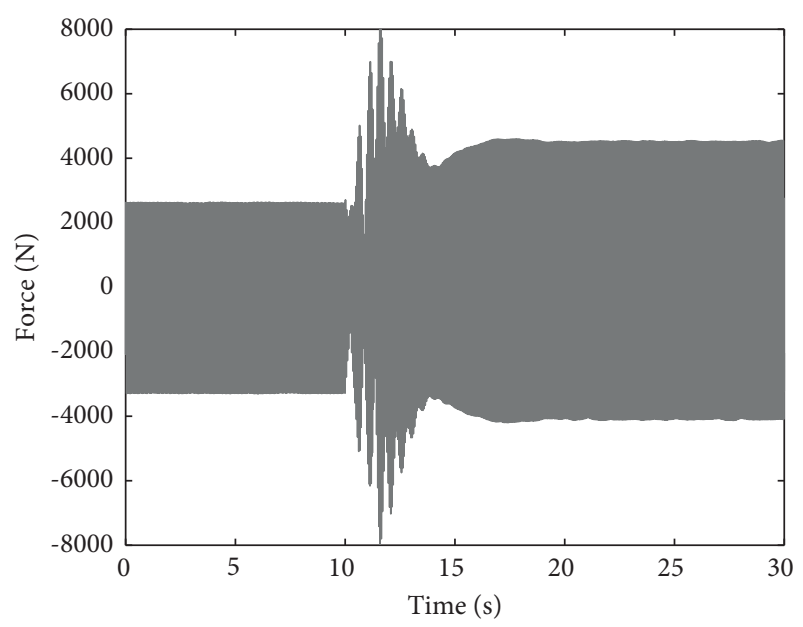

(b)

FIgURE 13: Acceleration response and filter output with LMS-MSRC algorithm. (a) Acceleration response at the control point. (b) Output of the filter.

changed slightly at $80 \mathrm{~s}$, and the amplitude of the error signal fluctuates more at $160 \mathrm{~s}$ and $240 \mathrm{~s}$ with LMS-MSRC. In comparison, multichannel LMS fluctuates greatly, and it takes more time to converge under phase changes. As is shown in Figure 17(c), excitation phase change is as high as $-75^{\circ}$ at $160 \mathrm{~s}$, and instantaneous change of the filter output increases, but the acceleration response is restored to the level before excitation phase fluctuation after a few seconds of adjustment. The results illustrate that LMS-MSRC hybrid controller exhibits adaptability to the disturbance phase mutation.
4.2.3. Time-Varying Primary/Secondary Path with Mutation in Disturbance Frequency and Amplitude. An additional $1 \mathrm{~kg}$ mass is placed between the PSA installation position and the controlled point before test. At about 80s, disturbance frequency turns to $18.5 \mathrm{~Hz}+37 \mathrm{~Hz}$ and amplitude increases by $50 \%$, with the mass removed to realize primary/ secondary path sudden changes of the elastic beam.

As is shown in Figures 18(a)-18(b), vibration attenuation is achieved before structural mutation with LMS-MSRC and multichannel LMS. However, due to the mass movement and the sharp increase in disturbance amplitude, the 


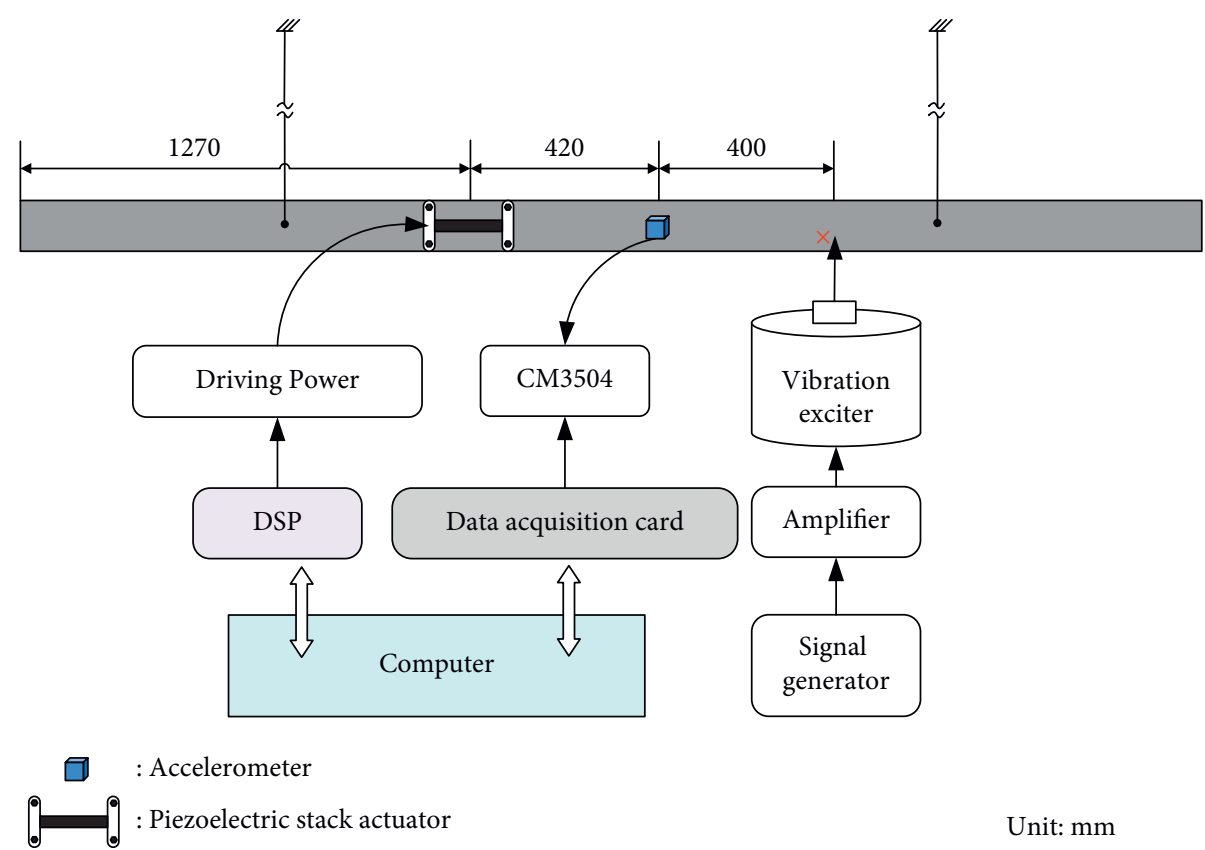

FIgURE 14: Schematic diagram of the elastic beam ACSR test system.

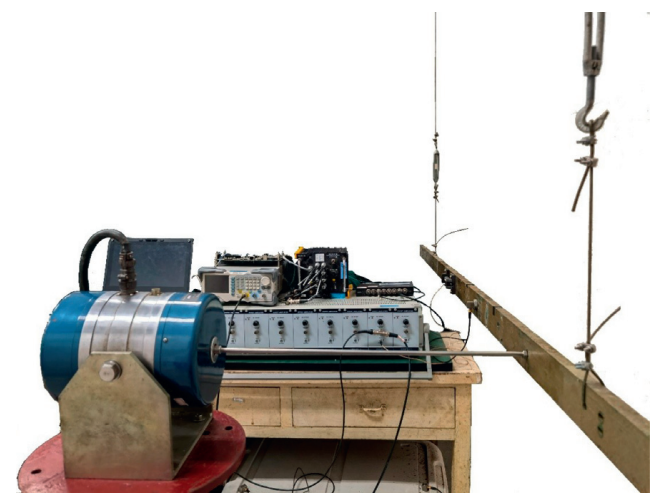

Figure 15: Physical diagram of the elastic beam ACSR experimental setup.

TABLE 1: Parameters of the beam structure.

\begin{tabular}{lc}
\hline Parameters & Data \\
\hline Length $\times$ width $\times$ height $(\mathrm{mm})$ & $3000 \times 60 \times 20$ \\
First three natural frequencies $(\mathrm{Hz})$ & $11.9,35,61$ \\
Control point $(\mathrm{mm})$ & 1690 \\
Actuator location $(\mathrm{mm})$ & 1270 \\
Exciter location $(\mathrm{mm})$ & 2090 \\
\hline
\end{tabular}

acceleration response increases rapidly. Figure 18(c) indicates that the instantaneous response overshoot is $42 \%$ less than the peak value, which demonstrates that additional damping is provided to effectively suppress response surge. In addition, the vibration attenuation reaches $85 \%$ within 2.5 s so as to verify the fast convergence and robustness of the algorithm. In contrast, it is observed that the response peak of multichannel LMS is the same as that of the open-loop system and the response is decreased by $83 \%$ within $20 \mathrm{~s}$ in Figure $18(\mathrm{~b})$. The
TABle 2: Parameters of the piezoelectric stack.

\begin{tabular}{lc}
\hline Parameters & Data \\
\hline Peak-to-peak displacement $(\mu \mathrm{m})$ & 100 \\
Axial stiffness $(\mathrm{N} / \mu \mathrm{m})$ & 20 \\
Blocked force $(\mathrm{N})$ & 3500 \\
First axial natural frequency $(\mathrm{kHz})$ & 10 \\
Diameter $\times$ length $(\mathrm{mm})$ & $15 \times 100$ \\
Input range $(\mathrm{V})$ & $0 \sim 100$ \\
\hline
\end{tabular}

acceleration response before and after excitation frequency change with LMS-MSRC is compared in Figure $18(\mathrm{~d})$. Although the $18.5 \mathrm{~Hz}$ and $37 \mathrm{~Hz}$ components are almost completely suppressed, the residual vibration increases. Analysing the response before and after the mass movement, the uncontrolled structure response caused by the movement is the main reason accounting for the increase of residual signal. The LMS-MSRC filter output in Figure 18(e) exhibits that the excitation 


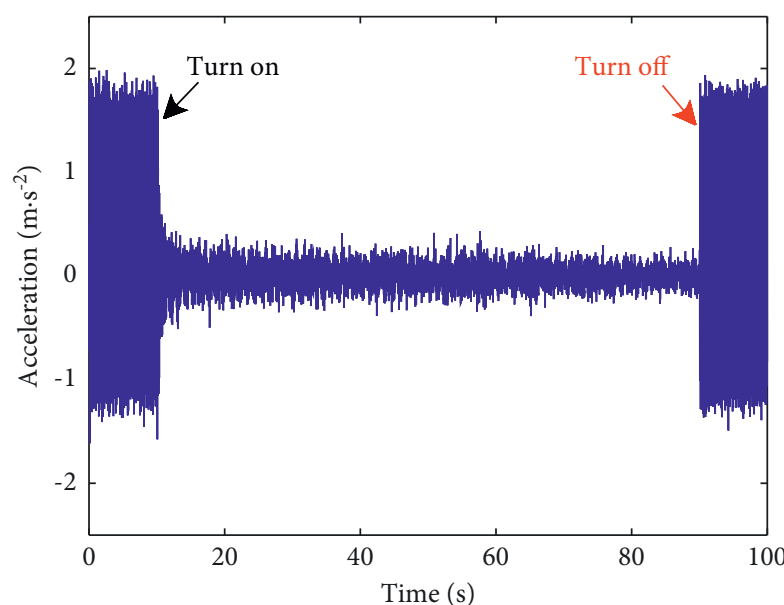

(a)

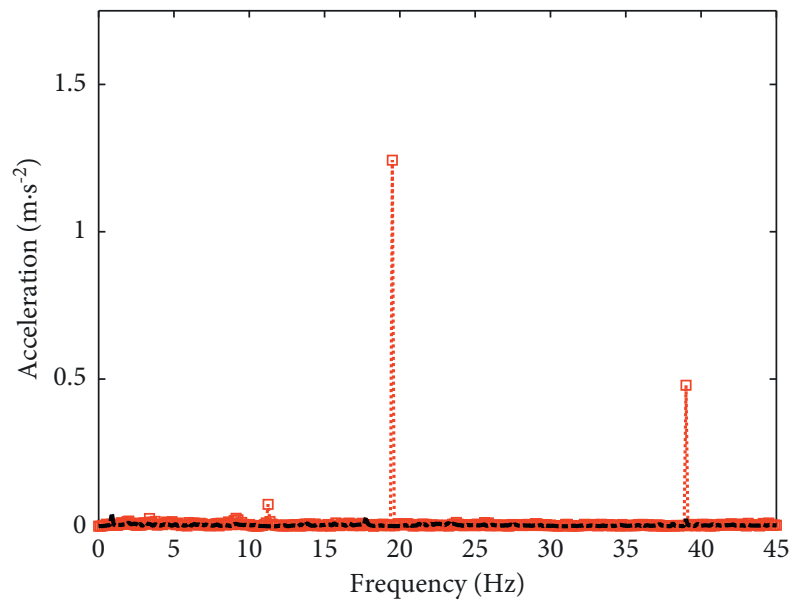

....... Turn off

-.-. Turn on

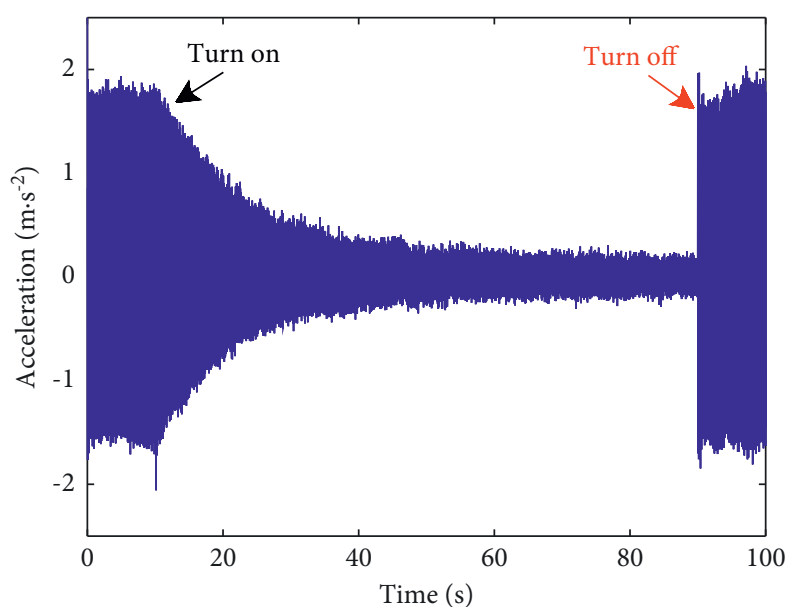

(b)

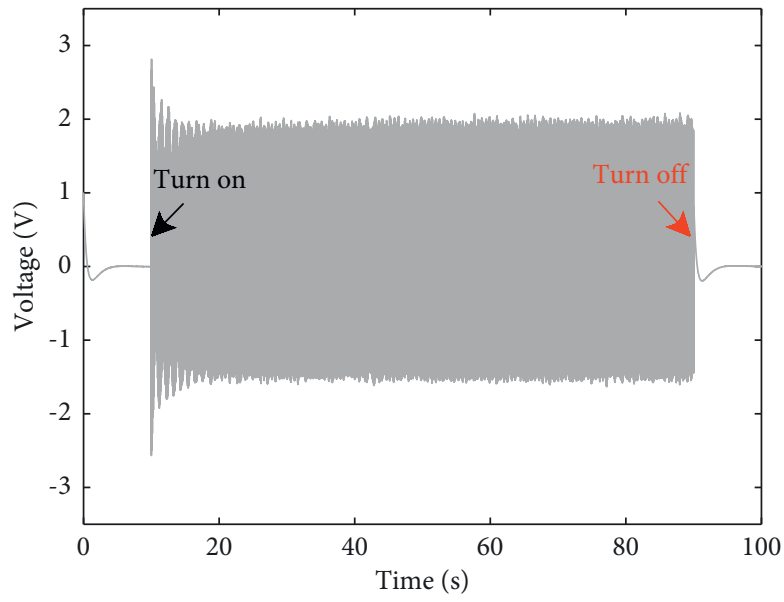

(d)

FIGURE 16: Acceleration response comparison and filter output with LMS-MSRC algorithm. (a) Acceleration response with LMS-MSRC. (b) Acceleration response with multichannel LMS. (c) Comparison of LMS-MSRC response spectrum. (d) Output of LMS-MSRC filter.

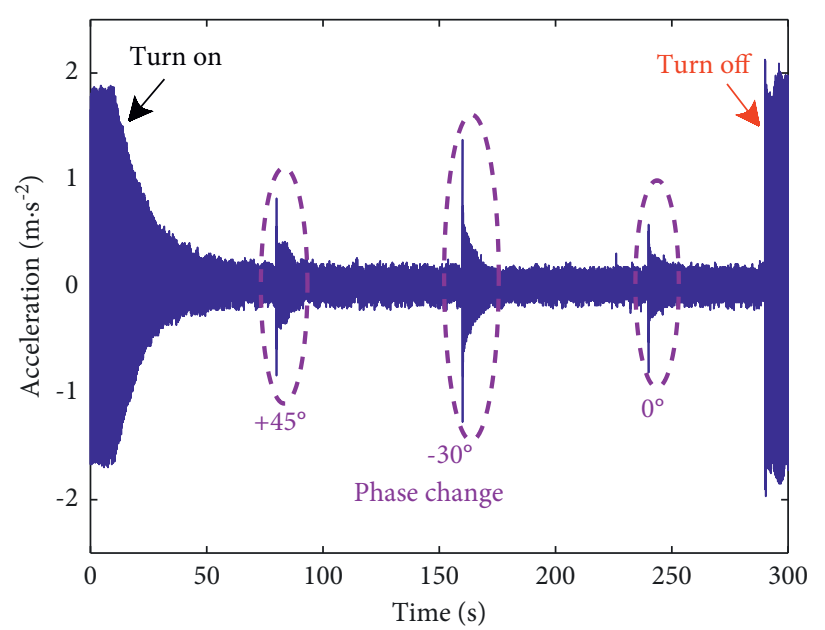

(a)

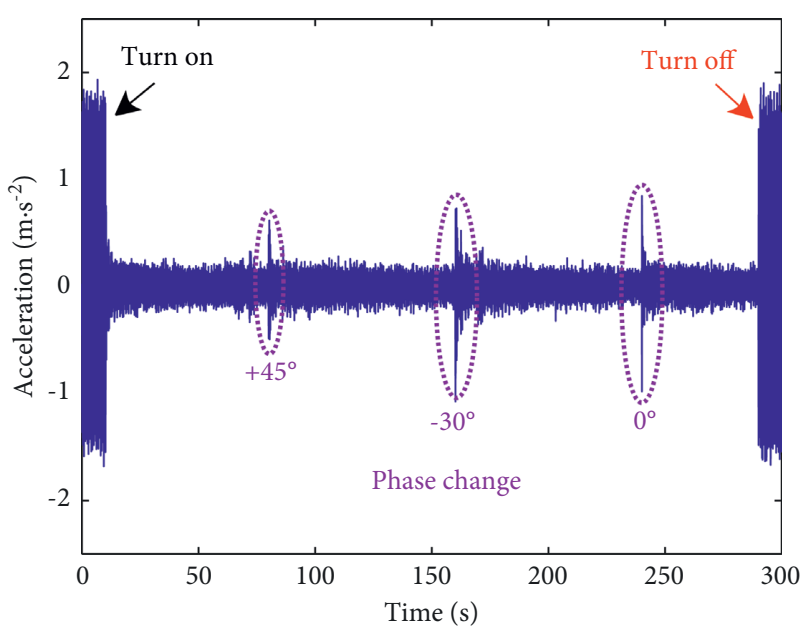

(b)

Figure 17: Continued. 


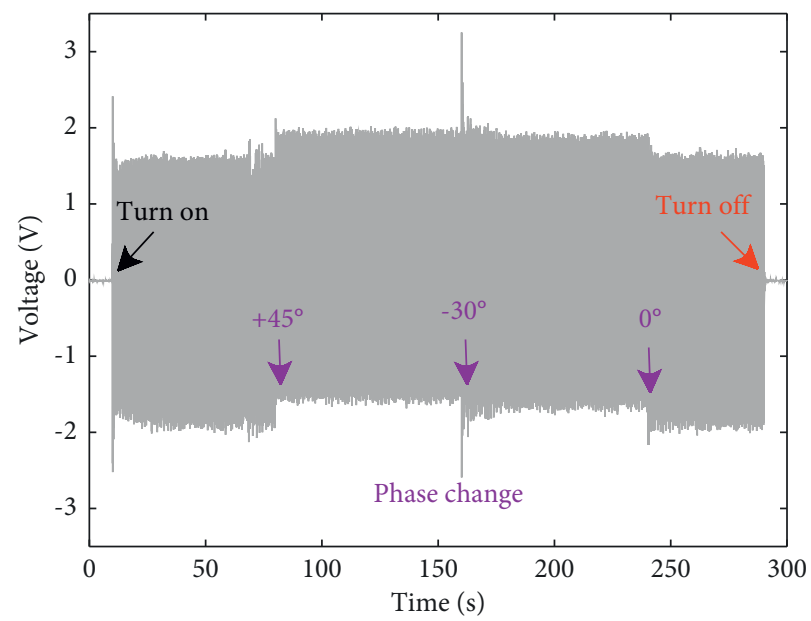

(c)

Figure 17: Control effects after disturbance phase changes. (a) Acceleration response with LMS-MSRC. (b) Acceleration response with multichannel LMS. (c) Output of LMS-MSRC filter.

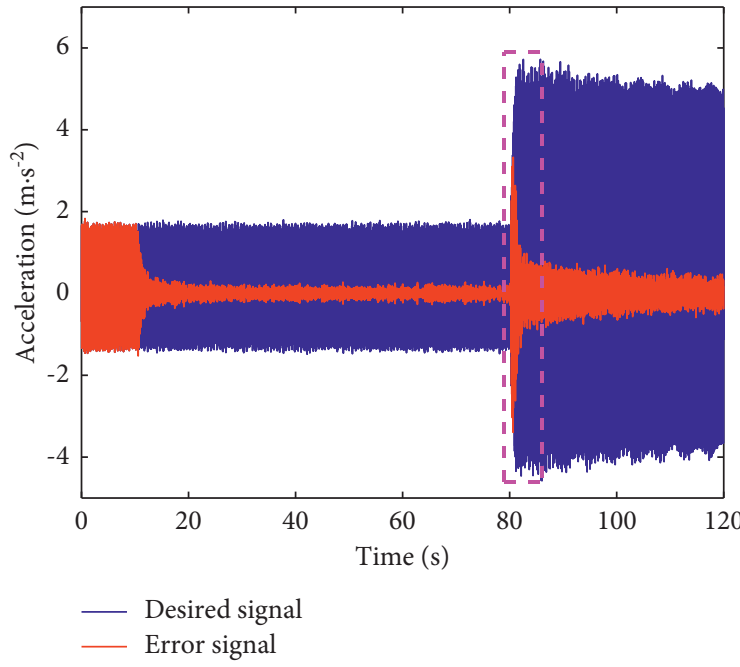

(a)

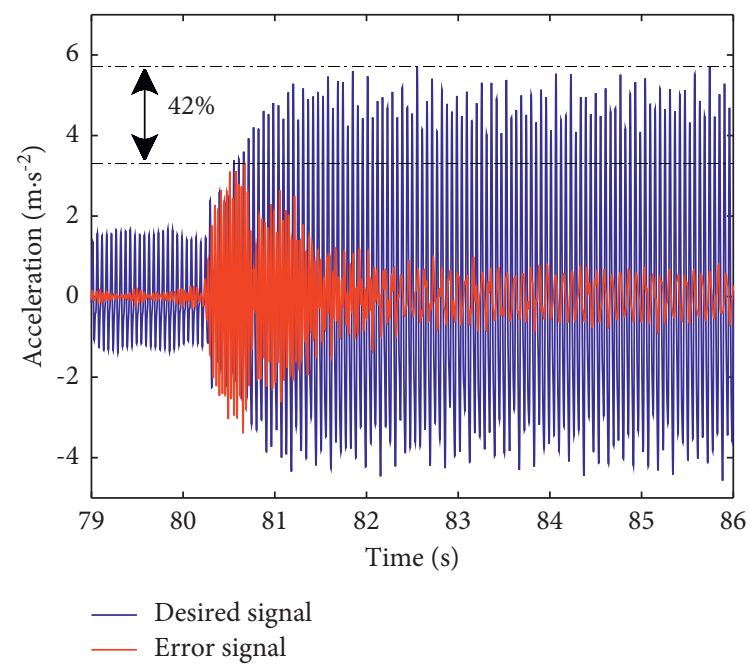

(c)

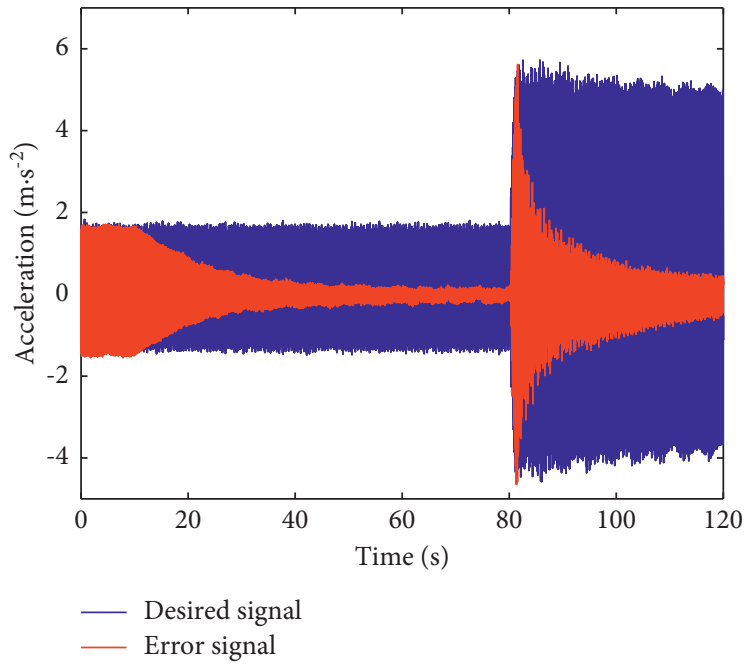

(b)

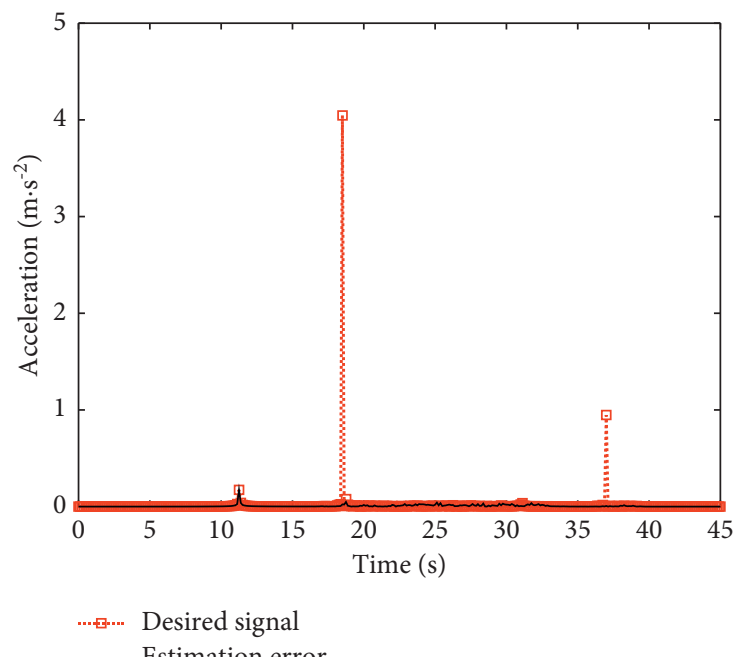

(d)

FIgURE 18: Continued. 


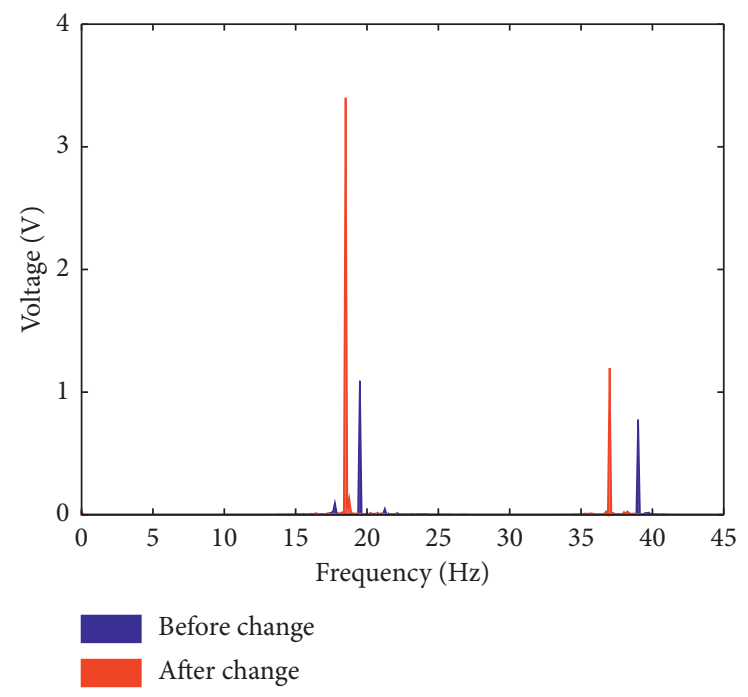

(e)

FIGURE 18: Control effects before/after disturbance and primary/secondary path mutation. (a) Acceleration response with LMS-MSRC. (b) Acceleration response with multichannel LMS. (c) Control process at mutation with LMS-MSRC. (d) Comparison of LMS-MSRC response spectrum. (e) Comparison of LMS-MSRC filter output spectrum.

frequencies decrease, but the uncontrolled response raises, resulting in an increase in the required control energy.

From the above experimental results, compared to multichannel LMS, LMS-MSRC based on reference signal reconstruction can effectively suppress multifrequency harmonic response and show good adaptability and strong robustness to unknown environmental changes. The fast-changing control signal avoids violent fluctuation of most structural response, and the mutation response is effectively suppressed during helicopter maneuvering flight.

\section{Conclusions}

For the purpose of helicopter ACSR during maneuvering flight, based on Fx-LMS algorithm, the reference signal reconstruction and the mixed sensitivity robust feedback controller are considered in this paper to form a feedforward-robust hybrid ACSR control law. In view of the simplified finite element helicopter model, several control simulations of typical helicopter maneuvering flight conditions are carried out. A series of ACSR tests to simulate helicopter maneuvering flight are conducted on a free-free beam. The main conclusions of the present study are summarized as follows:

(1) The designed robust feedback controller can make the hybrid control law obtain the advantages of feedforward and feedback control at the same time. That is, the feedback control can reduce the overall response and suppress unknown transient disturbance, while residual vibration related to the reference signal is cancelled by feedforward control so as to improve the global vibration attenuation during helicopter maneuvering flight. In addition, the introduction of the reconstructed reference signal can effectively track the real-time changes of the uncontrolled response, which is beneficial for the convergence and stability of the system.

(2) In the cases of complex transient external disturbance, time-varying secondary path, and changes in excitation signal amplitude and frequencies of the simulation object, LMS-MSRC based on reference signal reconstruction can effectively suppress multifrequency vibration, which shows obvious advantages in adaptive adjustment performance, robustness, and convergence speed compared with other ACSR methods. In the case of simulating the complex operating conditions of helicopter maneuvering flight, the introduction of robust feedback controller increases secondary path damping, and the response overshoot suppressing is achieved. The reconstructed reference signal that reflects the realtime change of disturbance accelerates convergence speed and enhances the robustness of the entire control system.

(3) In the ACSR tests on the free-free beam, the multifrequency vibration suppression performance and adaptability of LMS-MSRC are verified. Under the circumstances that external excitation amplitude, frequency, and primary/secondary path change simultaneously, the peak value of the closed-loop system is $42 \%$ lower than that of the open-loop system, and the vibration response is attenuated by more than $85 \%$ within 2.5 s. The experimental results demonstrate that LMS-MSRC based on reference signal reconstruction shows good adaptability and strong robustness to the unknown environmental changes with great application potential to helicopter ACSR during maneuvering flight. 


\section{Data Availability}

The data supporting the findings of the study cannot be shared at this time as the data also form part of an ongoing study.

\section{Conflicts of Interest}

The authors declare that they have no conflicts of interest regarding the publication of this paper.

\section{Acknowledgments}

The authors would like to thank the National Key Laboratory Foundation of China (no. 6142220040204) and the National Key Laboratory of Rotorcraft Aeromechanics.

\section{References}

[1] W. Johnson, Helicopter Theory, Dover Publications," Inc., New York, NY, USA, 1994.

[2] A. E. Staple and D. M. Wells, "The development and testing of an active control of structural response system for the EH-101 helicopter," in Proceedings of the 16th European Rotorcraft Forum, pp. 611-6111, Amsterdam, Netherlands, September 1990.

[3] R. K. Goodman and T. A. Millott, "Design, development and flight test of the active control system for the Sikorsky S-92," in Proceedings of the American Helicopter Society 56th Annual Forum, pp. 764-771, Virginia Beach, VA, USA, May 2000.

[4] B. Vignal and T. Krysinski, "Development and qualification of active vibration control system for the Eurocopter EC225/ EC725," in Proceedings of the American Helicopter Society 61st Annual Forum, pp. 96-106, Grapevine, TX, USA, June 2005.

[5] T. A. Millot, J. K. Goodman, J. K. Wong, W. A. Welsh, J. R. Correia, and C. E. Cassil, "Risk reduction flight test of a pre-production active vibration control system for the UH60M," in Proceedings of the American Helicopter Society 59th Annual Forum, pp. 496-505, Phoenix, AZ, USA, May 2003.

[6] L. Pan and R. Chen, "Formulation and validation of a helicopter model for pull-up maneuver simulation," Acta Aeronautica et Astronautica Sinica, vol. 31, no. 12, pp. 2315-2323, 2010.

[7] M. J. Bhagwat, R. A. Ormiston, H. A. Saberi, and H. Xin, "Application of CFD/CSD coupling for analysis of rotorcraft airloads and blade loads in maneuvering flight," in Proceedings of the American Helicopter Society 63rd Annual Forum, Virginia Beach, VA, USA, May 2007.

[8] M. Edward, I. Y. Huff, Tumer, and E. Barszcz, "An analysis of maneuvering effects on transmission vibrations in an AH1 Cobra Helicopter," Journal of the American Helicopter Society, vol. 47, 2012.

[9] U. T. P. Arnold and D. Fürst, "Closed loop IBC results from CH-53G flight tests," Aerospace Science and Technology, vol. 9, no. 5, pp. 421-435, 2005.

[10] A. V. Popov, "Proof-of-match technique for Bell 427 helicopter level D simulator," Masters Abstracts International, vol. 44, no. 4, 2005.

[11] R. S. Mahmood and D. Heverly, "In-flight demonstration of active vibration control technologies on the bell 429 helicopter," in Proceedings of the American Helicopter Society 68th Annual Forum, Fort Worth, TX, USA, May 2012.
[12] D. H. Kim, D. I. Kwak, and Q. Song, "Demonstration of active vibration control system on a Korean utility helicopter," International Journal of Aeronautical and Space Sciences, vol. 20, 2018.

[13] F. K. Straub, V. R. Anand, T. S. Birchette, and B. H. Lanu, "SMART rotor development and wind tunnel test," in Proceedings of the 35th European Rotorcraft Forum, pp. 413-430, New York, NY, USA, September 2009.

[14] D. Heverly, K. W. Wang, and E. C. Smith, Optimal actuator placement and active structure design for control of helicopter airframe vibrations, Ph.D. thesis, Pennsylvania State University, State College, PA, USA, 2002.

[15] S. R. Viswamurthy and R. Ganguli, "Effect of piezoelectric hysteresis on helicopter vibration control using trailing-edge flaps," Journal of Guidance, Control, and Dynamics, vol. 29, no. 5, pp. 1201-1209, 2006.

[16] H. John, S. Doug, and B. A. Askari, "Vibration attenuation through the use of active frahms," in Proceedings of the 46th Annual Forum Of the American Helicopter Society, Phoenix, AZ, USA, May 2003.

[17] D. Patt, L. Liu, J. Chandrasekar, D. S. Bernstein, and P. P. Friedmann, "Higher-harmonic-control algorithm for helicopter vibration reduction revisited," Journal of Guidance, Control, and Dynamics, vol. 28, no. 5, pp. 918-930, 2005.

[18] W. Johnson, "Self-tuning regulators for multi-cyclic control of helicopter vibrations," NASA Ames Research Center, Moffett Field, CA, USA, NASA Technical Paper, 1982.

[19] C. F. Zhao, "Study on active control of structural responses for helicopter in frequency domain," Master thesis, Nanjing University of Aeronautics and Astronautics, Nanjing, China, 2010.

[20] L. S. Song and P. Q. Xia, "A harmonic synchronous identification-updating method for active control of helicopter structural response driven by piezoelectric stack actuators," Journal of the American Helicopter Society, vol. 60, 2015.

[21] D. Meng, P. Xia, and L. S. Song, "MIMOMH feed-forward adaptive vibration control of helicopter fuselage by using piezoelectric stack actuators," Journal of Vibration and Control, vol. 24, 2018.

[22] L. Zhang and X. Qiu, "Causality study on a feedforward active noise control headset with different noise coming directions in free field," Applied Acoustics, vol. 80, no. 5, pp. 36-44, 2014.

[23] F. Hoffmann, R. Maier, P. Janker, and F. Hermle, "Helicopter interior noise reduction by using active gearbox struts," in Proceedings of the 12th AIAA/CEAS Aeroacoustics Conference, Cambridge, MA, USA, May 2006.

[24] M. Li, T. C. Lim, W. S. Shepard, and Y. H. Guan, "Experimental active vibration control of gear mesh harmonics in a power recirculation gearbox system using a piezoelectric stack actuator," Smart Materials and Structures, vol. 14, no. 14, p. 917, 2005.

[25] S. D. Snyder and C. H. Hansen, "The effect of transfer function estimation errors on the filtered-x LMS algorithm," IEEE Transactions on Signal Processing, vol. 42, no. 4, pp. 950-953, 1994.

[26] S. Skogestad and I. Postlethwaite, Multivariable Feedback Control: Analysis and Design, John Wiley \& Sons, Hoboken, NJ, USA, 2005.

[27] R. M. Howard, Linear System Theory, Wiley-IEEE Press, Hoboken, NJ, USA, 2002.

[28] K. Chen, Active Noise Control, National Defense Industry Press, Beijing, China, 2nd edition, 2014.

[29] P. Diniz, Adaptive Filtering: Algorithms and Practical Implementation, Springer, Berlin, Germany, 2008. 
[30] O. A. Bauchau, J. Rodriguez, and S.-Y. Chen, "Coupled rotorfuselage analysis with finite motions using component mode synthesis," Journal of the American Helicopter Society, vol. 49, no. 2, pp. 201-211, 2004.

[31] M. Y. Lu, Adaptive predictive control of structural responses for helicopter, PhD thesis, Nanjing University of Aeronautics and Astronautics, Nanjing, China, 2005. 